\title{
Variations of the orbital periods in semi-detached binary stars with radiative outer layers
}

\author{
V. Šimon \\ Astronomical Institute, Academy of Sciences, 25165 Ondřejov, Czech Republic \\ Received January 29; accepted June 23, 1998
}

\begin{abstract}
A detailed analysis of the period changes of sixteen semi-detached binaries which contain only stars with radiative outer layers (ET-systems) has been performed and their respective $\mathrm{O}-\mathrm{C}$ diagrams are brought and discussed. It was found that the course of the period variations is monotonic and in some systems the period is even constant. This detailed analysis of extensive sets of timings covering several decades brings a strong support to an earlier finding of Hall (1989) and develops it further. We show that all systems with the orbital periods shorter than nine days display constant period or its increase, with the exception of an uncertain case of V 337 Aql. The course of the period variations in TT Aur appears more complex but the cyclic term can be plausibly explained by the third body.

A search for general relations between the parameters of the systems and the period changes was undertaken. The mass ratio $q$ appears to play a role in the period variations. Clear changes in systems with orbital periods shorter than 9 days were detected only for $q>0.4$ while constant periods are common in systems with $q<0.4$ in this period range. The sense of the secular changes in most systems is in accordance with the dominant conservative mass transfer in evolved binary and corresponds to the slow phase. The role of the evolutionary scenarios (case A versus B) and the influence of changes of the rotational angular momenta of the components are discussed.
\end{abstract}

Key words: stars: activity — binaries: eclipsing binaries: close - circumstellar matter — stars: earlytype - stars: evolution

\section{Introduction}

Changes of the orbital periods are well documented for a large number of close eclipsing binaries of various types.

Send offprint requests to: V. Šimon: simon@sunstel.asu.cas.cz
There were developed several theories for their explanation which employ two categories of causes: the geometric and the intrinsic ones. The geometric ones comprise the apsidal motion and the presence of the third body (lighttime effect, LITE). They require strict periodicity of the changes which course can be analytically expressed.

Nevertheless, courses of most period changes are nonperiodic and cannot be explained by the geometric effects. Already Wood (1950) recognized that systems with large period changes contain at least one star which fills in its Roche lobe. As Huang (1963) showed period changes can be consequences of mass transfer in binary or mass loss from it. Also models of mass exchange in binary which were developed in sixties and early seventies seemed to offer a natural explanation for some period changes and showed the importance of the semi-detached configuration (e.g. Paczynski 1967; Plavec 1968).

Most following analyses were done for Algols, the typical representatives of the semi-detached binaries with evolved late-type star filling in its Roche lobe and transferring mass onto its companion, a main-sequence star of early spectral type. Analyses of the long series of observations revealed that period changes in a large part of Algols are much more complicated than the evolutionary schemes of mass transfer suggested and alternate course of variations is not exceptional (e.g. U Cep-Fig. 1 in Olson et al. 1981). Biermann \& Hall (1973) presented a model which tried to explain this phenomenon. They assumed that the mass is transferred in bursts and the angular momentum is temporarily stored in the outer layer of the gainer, thus causing decrease of the period. In the subsequent epoch the increased turbulence in the gainer allows to return this angular momentum to the orbit and the orbital period increases again.

A significant change in opinions on the mechanisms of the period variations occurred in the eighties. An idea appeared that the mass transfer rate is too small to be important for the observed changes. Matese \& Whitmire (1983) described a general mechanism which explains the 
observed period variations by changes of the stellar structure. Hall (1989) presented a correlation between spectral types of the binary components and character of the period changes. His statistics showed that binaries which contain at least one star of spectral type F5 or later show cyclic course of the $\mathrm{O}-\mathrm{C}$ variations while only monotonic course or constant period is observed in systems with purely early-type components. Hall attributed the cyclic course to the magnetic activity of the late-type star possessing convective outer layer (COL). Applegate (1992) and Richman et al. (1994) presented a theory which explains the cyclic orbital period variations by changes of the internal structure, caused by the sub-surface magnetic field in COL of a component of the binary. This theory forecasts that changes of the orbital period should be accompanied by the luminosity variations of this star through the cycle and thus allow for an observational test. Hall (1991) found that the period of CG Cyg, an RS CVn-type system consisting of two main sequence stars $(\mathrm{G} 9.5 \mathrm{~V}+\mathrm{K} 3 \mathrm{~V})$, varies with the cycle-length of 50 years and these changes are accompanied by variations of brightness in agreement with the Applegate theory. Maximum brightness and period increase occurred simultaneously. On the contrary, Šimon (1997c) revealed two consecutive episodes of shortening the orbital period in the Algol-type system RW Tau. Each event was accompanied by decrease of brightness of the loser. The course of the changes in case of RW Tau was inconsistent with the model proposed by Applegate (1992).

In summary, the evidences were accumulated which suggest that the late-type component is responsible for most observed period changes. An important question therefore arises: what will remain if we remove the suggested effects of the late-type component? If the supposed dominant influence of this star is avoided one could also have a better chance to evaluate the role of the mass transfer in the period changes. Further, if the latest sets of parameters of the respective systems are employed, it is possible to assess their role in the variations of the period. The following analysis which preliminary version was presented by Šimon (1997ab) will try to bring some answers.

\section{General considerations}

In order to avoid influence of the late-type component, period changes of binaries in which both stars are of early spectral types must be analysed. COL is supposed to appear at F5 in single stars. Since losers in binaries undergoing mass outflow may develop deeper COL than single stars of the same spectral type we will restrict to binaries with both stars earlier than F0.

The following analysis will be devoted to the Algoltype semi-detached systems because we can make use some of their suitable features: (a) their status is forecasted by models and they represent a rather well understood epoch of evolution of close binaries; (b) mass transfer in this binary can occur only in one well defined sense, i.e. from the Roche-lobe filling component (loser); (c) in principle, the flowing matter can be observed since the gainer may be well inside its Roche lobe; $(d)$ the mass transfer or loss rate can be determined from spectroscopy or photometry independently of the analysis of changes of the orbital period in some cases.

The early-type binaries which are a matter of this study contain high-mass stars. Since these stars evolve more rapidly than those with smaller masses we can also expect more pronounced evidences for mass transfer events and evolutionary processes. Although the available timings cover a limited interval (usually a century or shorter) which may be too short for the convincing changes produced by the mass transfer to manifest themselves even in the high-mass binaries this handicap can be at least partly overcome by simultaneous analysis of a set of systems and by a search for common features. The activity of the cool component is supposed to be responsible for most period changes in Algols. The $\mathrm{O}-\mathrm{C}$ variations in the early-type binaries which should be free of this activity could then allow for a better insight to the importance of the mass transfer for the period changes, especially in those cases where an independent derivation of the mass transfer rate from the photometric or spectroscopic activity is possible.

Nevertheless, some reasons for caution should be noted. The number of the observable high-mass eclipsing binaries with an early-type star filling its Roche lobe (hereafter ET-systems) is significantly smaller than that of Algols with late-type losers (hereafter LL-Algols). This also implies a small number of ET-systems with the well determined parameters. ET-systems also generally display shallower eclipses because the luminosities of both components are more similar to each other than in LL-Algols. Accurate timings are therefore more difficult especially for visual observers.

\section{Sources of the data and analysis of the O-C graphs}

An extensive search for ET-systems was undertaken in the literature. Only systems for which solutions of the light curve giving the semi-detached configuration (or very close to it) and preferably also the spectroscopic observations exist were used for the following analysis. The number of the suitable systems was also inevitably lowered by the requirement of a good coverage by the timings amounting several decades. The parameters of the final ensemble of binaries are summarized in Table 1 where also the sources are cited. The radii of the gainers in $\beta$ Lyr and RY Sct, supposed to be veiled by the accretion disk, were obtained by an interpolation in the table given by Harmanec (1988). These radii were determined for the masses and inferred spectral types given in the cited papers.

The international database of timings of minima of the eclipsing binaries founded by Mr. Lichtenknecker and 
Table 1. The parameters of binaries with early-type components used for this analysis. The effective temperatures of the primary (gainer) and secondary (loser) component are given in the columns $T_{\text {eff }}(1)$ and $T_{\text {eff }}(2)$, respectively. The mass and radius of the gainer are denoted as $M_{1}, R_{1}$ while those of the loser are marked by $M_{2}$ and $R_{2}$. These values are in solar units. The mass ratio $q$ is calculated as $M_{2} / M_{1}$. The orbital separation $a$ (in solar radii) is given along with the fractional radius of the primary $R_{1} / a$

\begin{tabular}{lrrrrrrrrlll}
\hline Binary & Period (d) & $T_{\text {eff }}(1)$ & $T_{\text {eff }}(2)$ & $M_{1}$ & $R_{1}$ & $M_{2}$ & $R_{2}$ & $q$ & $a$ & $R_{1} / a$ & Ref. \\
\hline V337 Aql & 2.734 & 25000 & 21800 & 17.0 & 9.4 & 10.0 & 8.3 & 0.6 & 24.7 & 0.374 & 1,13 \\
SX Aur & 1.210 & 25000 & 18900 & 10.30 & 5.18 & 5.60 & 3.90 & 0.544 & 12.01 & 0.4313 & 2 \\
TT Aur & 1.333 & 24800 & 18000 & 8.10 & 3.90 & 5.40 & 4.20 & 0.667 & 12.13 & 0.3215 & 3 \\
BF Aur & 1.583 & 16000 & 15970 & 4.8 & 4.2 & 5.0 & 4.7 & 1.05 & 12.23 & 0.3434 & 4 \\
AQ Cas & 11.721 & 28000 & 12820 & 16.6 & 7.55 & 4.72 & 16.6 & 0.284 & 60.19 & 0.1254 & 5 \\
XZ Cep & 5.097 & 30000 & 23120 & 15.8 & 7.00 & 6.4 & 10.5 & 0.405 & 35.11 & 0.1994 & 6 \\
V 448 Cyg & 6.520 & 30000 & 20460 & 25.2 & 6.7 & 14.0 & 16.3 & 0.556 & 50.00 & 0.1340 & 6 \\
u Her & 2.051 & 20000 & 11600 & 7.60 & 5.79 & 2.90 & 4.40 & 0.382 & 14.87 & 0.3894 & 2 \\
VY Lac & 1.036 & 12000 & 9170 & 4.3 & 3.1 & 2.6 & 2.8 & 0.603 & 8.22 & 0.378 & 7 \\
$\beta$ Lyr & 12.914 & & 13300 & 14.1 & 6.1 & 4.25 & 16.7 & 0.301 & 61.10 & 0.100 & 8 \\
DM Per & 2.728 & 18000 & 8400 & 7.31 & 4.50 & 2.07 & 4.75 & 0.283 & 17.32 & 0.2598 & 3 \\
IZ Per & 3.688 & 17250 & 8740 & & & & & 0.36 & & 0.323 & 9 \\
RY Sct & 11.124 & & B0 & 26.0 & 8.0 & 8.0 & 20.4 & 0.301 & 67.5 & 0.1185 & 12 \\
RZ Sct & 15.191 & 18000 & 6420 & 11.7 & 15.8 & 2.49 & 15.9 & 0.213 & 62.46 & 0.2530 & 10 \\
V356 Sgr & 8.896 & 16500 & 8600 & 12.1 & 6.0 & 4.7 & 14.0 & 0.388 & 46.25 & 0.1297 & 11 \\
Z Vul & 2.455 & 18000 & 9020 & 5.40 & 4.70 & 2.30 & 4.50 & 0.426 & 15.12 & 0.3108 & 2 \\
\hline
\end{tabular}

References: 1. Giuricin \& Mardirossian (1981a); 2. Hilditch \& Bell (1987); 3. Figueiredo et al. (1994); 4. Kallrath \& Kamper (1992); 5. Olson (1994); 6. Harries et al. (1997); 7. Semeniuk \& Kaluzny (1984); 8. Harmanec (1990); 9. Wolf \& West (1993); 10. Olson \& Ezel (1994); 11. Popper (1980); 12. Skulskij (1992); 13. Alduseva (1977).

now directed by F. Agerer was the main source of timings used for this analysis (Agerer 1995, 1996). The O-C values were calculated for the timings of each binary according to the ephemerides found in the literature in the first step (usually SAC No. 67, Danielkiewicz- Krosniak and Kurpinska- Winiarska 1996, hereafter SAC96). Since the timings were obtained by various methods the widely accepted weights (photoel. $=10$, photographic $=3$, visual $=$ 1) were attributed to the single timings. The $\mathrm{O}-\mathrm{C}$ graphs were submitted to a visual inspection. In some cases when a large amount of visual minima was available these timings were grouped into bins of several observations and averaged. Such means were given higher weights. Several largely deviating minima were rejected from the sets.

Errors of the photoel. timings are usually smaller than the symbols used and therefore do not represent serious problem. Unfortunately, the accuracies of the visual and photographic timings, generally having larger scatter, are not available in many cases. One possible way how to assess them is a visual inspection of the $\mathrm{O}-\mathrm{C}$ diagrams. As can be seen from the attached figures most these data are in a good agreement with the photoelectric ones. Accuracy of the visual and photographic timings was further evaluated by linear fits of these data inside the well covered segments of the $\mathrm{O}-\mathrm{C}$ curves where the period $P$ could be approximated as roughly constant. The $1 \sigma$ errors of these timings were then determined from the residuals of the fit using the least squares method.

In general, the course of the $\mathrm{O}-\mathrm{C}$ changes is often complicated and it is not possible to give any unique method for their analysis. An overview and discussion of the exist- ing methods and general considerations of analysis of an O-C diagram can be found in Šimon (1997c). The visibility of the period change becomes considerably suppressed with the growing slope of the $\mathrm{O}-\mathrm{C}$ curve on the plot. The period lengths were therefore calculated to give the slope close to zero in a large part of the curve. The photoelectric observations which well define the $\mathrm{O}-\mathrm{C}$ curve are usually found in the second half of the interval covered by the timings in the systems analysed here. In this case it often appears advantageous to keep the slope of these photoelectric data close to zero since the eventual period change (namely if it is inferred from the old timings obtained by other methods) can be more easily resolved and evaluated.

We admit that indication of the period change in a given binary is dependent on the chosen criteria to some extent. We will consider the period change in a given binary as present if it can be resolved by the visual inspection of the $\mathrm{O}-\mathrm{C}$ diagram which is constructed according to the principles listed above. Its course must be defined by multiple observations. Polynomial fits to the $\mathrm{O}-\mathrm{C}$ values (e.g. Kalimeris et al. 1994) appear to be a plausible method of analysis of the period change in our case. We note in advance that such fits to the $\mathrm{O}-\mathrm{C}$ values of the respective systems analysed here have shown that even the most variable periods could be plausibly matched by the second order polynomials. Comparison of the sum of the squares of the residuals of the linear $\left(C_{\mathrm{LIN}}\right)$ and parabolic $\left(C_{\mathrm{PAR}}\right)$ fits of the data can then serve as another clue in assessment of the period change. We will therefore introduce the ratio $S=C_{\mathrm{LIN}} / C_{\mathrm{PAR}}$; the larger $S$, the better defined variation. The results, discussed for the respective 
systems below, have shown that monotonic change of the $\mathrm{O}-\mathrm{C}$ values can be usually well recognized for $S=1.2$; this figure may be accepted as a typical limiting value above which the period can be regarded as variable. Discussions of the data and variability of the periods of the respective systems are given below.

The $\mathrm{O}-\mathrm{C}$ values in the diagrams plotted here are expressed in days as is usual. Nevertheless, it should be noted that the range of the period lengths in the respective systems analysed here is high and amounts about one to ten. The longer periods generally imply longer duration of the eclipse what leads to a larger scatter of the $\mathrm{O}-\mathrm{C}$ values. This scatter is more pronounced especially for the timings obtained by other methods than photoelectric. If the variations of the $\mathrm{O}-\mathrm{C}$ values are expressed as a fraction of period $P$ instead of days, eventually if the $\mathrm{O}-\mathrm{C}$ values are scaled down according to the respective period lengths of the systems then it can be shown that these values for the different systems usually display comparable scatter.

\section{The $\mathrm{O}-\mathrm{C}$ diagrams of the respective systems}

The analysis of the $\mathrm{O}-\mathrm{C}$ diagrams for the respective ETsystems listed in Table 1 is presented in this chapter. The $\mathrm{O}-\mathrm{C}$ diagrams for most of the systems are displayed to demonstrate the accuracy and reliability of the period changes, eventually the constancy of the orbital period.

Some binaries already known to display LITE (IU Aur - Mayer 1990) or seriously suspected of it (ZZ Cas - Kreiner \& Tremko 1993) were rejected from the set because this effect can often preclude visibility of the "intrinsic" changes which are the target of this analysis.

\section{1. $V 337 A q l$}

The latest solution of the light curve of this system comes from Giuricin \& Mardirossian (1981a) and is based on the mass ratio $q=0.6$ obtained from the RV measurements by Alduseva (1977). Catalano et al. (1971) reported variable light curve and possible decrease of the period length in the past. Mayer (1987) did not confirm the continuing decrease in the recent decades but admitted LITE.

The period given in SAC96 is too long. The new elements were determined and are given in Eq. (1). The $\mathrm{O}-\mathrm{C}$ values for the available timings calculated according to this ephemeris are displayed in Fig. 1. Standard deviation of the photographic data is 0.011 days.

$T(\min \mathrm{I})=2441168.401+2.733885534 E$.

There is a tentative suggestion of a decrease of the period length within the covered interval but this trend is to a large extent dependent on the old photographic timings. While some of these old timings of the primary minima

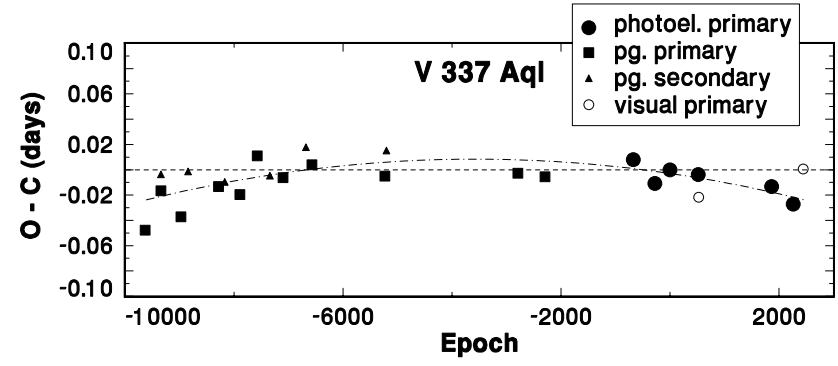

Fig. 1. The O-C diagram for V 337 Aql. The possible parabolic trend leading to a decrease of $P$ is marked

suggest longer period in the past the course of the secondary ones (probably not used in the previous analyses) may be consistent with the constant period. These photographic data (apart from one) come from a single source (Dugan \& Wright 1939). The photoelectric timings are available only for a short interval. A parabolic fit of all weighted $\mathrm{O}-\mathrm{C}$ values was attempted. The result is shown in Fig. 1 but it must be regarded as uncertain. It yields $\Delta P / P \approx-6.510^{-10}$ days $^{-1}$ with $S=1.9$. Although this value of $S$ is rather high, the evidence for a period decrease is based just on two old photographic timings and a single photoelectric observation, as can be inferred from Fig. 1. New observations are therefore urgently needed.

\section{2. $S X$ Aur}

This system was classified as semi-detached by Stothers (1973) but as he noted the components are very close to each other. This conclusion was confirmed by Bell et al. (1987). The gainer almost fills in its lobe (Fig. 17a) and according to Bell et al. is evolving into contact.

The elements given in SAC96 roughly satisfy the O-C values in the second half of the data set. The examination revealed that the $\mathrm{O}-\mathrm{C}$ values of the photoelectric timings spanning about 40 years can be well approximated by a straight line and allow for an improvement of the elements. The new ephemeris is given in Eq. (2) and was also used for the construction of the $\mathrm{O}-\mathrm{C}$ diagram in Fig. 2. Standard deviation of the photographic and visual data is 0.003 days.

$T(\min \mathrm{I})=2445739.5948+1.210080017 \mathrm{E}$.

It can be readily seen that the orbital period is variable and was definitely increasing during the last 82 years. Nevertheless, it is difficult to determine the exact course of this change. As was mentioned above the photoelectric data are consistent with the constant period in the last four decades. The parabolic fit of the whole data set which is displayed in Fig. 2 can plausibly match all the O-C data apart from one photoel. timing in $E=-10500$ which is displaced by about $-7 \mathrm{~min}$. The parabolic fit with $S=3.19$ yields the rate of the period change 


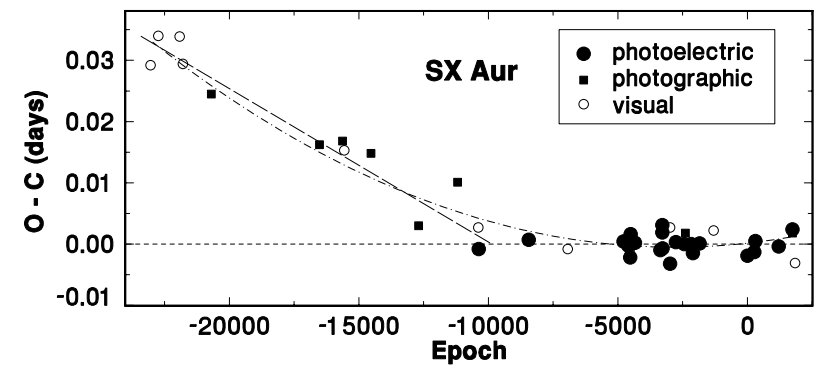

Fig. 2. The $\mathrm{O}-\mathrm{C}$ curve for $\mathrm{SX}$ Aur. The photoelectric data within $E=-11000$ to 1900 are consistent with the constant period while the older data suggest increase of $P$ inside the covered interval. Both parabolic fit of the whole data set and linear fit of the segment are displayed. See the text for details

$\Delta P / P=1.3610^{-10}$ days $^{-1}$ which is in a good agreement with the value determined by Kreiner \& Tremko (1978).

For the sake of completeness, a linear fit to a segment of the data within $E=-23050$ to -10000 was made (a long-dashed line in Fig. 2). The corresponding magnitude of the period change is $\Delta P / P=2.0710^{-6}$ days $^{-1}$ for this case. As can be seen in Fig. 2 the data do not allow to resolve which fit is more appropriate.

\subsection{TT Aur}

TT Aur is a close semi-detached system (Bell et al. 1987). An extensive set of timings obtained by various methods and covering 88 years exists for this binary. The $\mathrm{O}-\mathrm{C}$ values were calculated according to the elements given by Hanžl (1994). The visual inspection of the plot (Fig. 3) revealed a complicated course of the $\mathrm{O}-\mathrm{C}$ values. The photoelectric timings are available for the interval of $E=-10000$ to 1000 (36 years). The orbital period from Hanžl (1994) satisfies the mean course of O-C values in this interval but the moment of the basic minimum needs to be shifted by -0.0054 days (see below). The new ephemeris is given in Eq. (3). Even the photoelectric data display an unusually large scatter. A detailed examination of the plot of the $\mathrm{O}-\mathrm{C}$ values and consequent search for periodicity, carried out using PDM program (Stellingwerf 1978), revealed that this scatter is caused by cyclic variations on the time scale of about 12 years.

$T(\min \mathrm{I})=2448599.2964+1.332735 E$.

Although the cyclic trend can be traced also in the means of four visual timings (triangles in Fig. $3 ; \sigma=0.0047$ days) it was decided to base its analysis only on the set of thirteen photoelectric minima and one photographic timing since these changes are well defined there. The PDM program revealed two closely spaced periods: 4465 days (significance $\Theta=0.1762)$ and 4286 days $(\Theta=0.1773)$. The data used for this search therefore cover almost three consecutive cycles. The orbital solution found by the program SPEL showed that the $\mathrm{O}-\mathrm{C}$ changes are consistent with
Table 2. Orbital elements of the hypothetical third body in TT Aur determined from the photoelectric timings. $A$ is the semi-amplitude of the $\mathrm{O}-\mathrm{C}$ changes in days. $T_{0}$ is the systematic shift of the fitting. A rms error of one measurement of unit weight is $0.0008 \mathrm{~d}$

\begin{tabular}{lrrr}
\hline Period $P_{2}$ & $4465 \mathrm{~d}$ & $(12.2$ years $)$ \\
$T_{\max .}(\mathrm{O}-\mathrm{C})$ & $2443872 \pm$ & $245 \mathrm{JD}$ \\
$e$ & $0.296 \pm$ & 0.022 & \\
$\omega$ & $346.9^{\circ} \pm$ & $9.5^{\circ}$ & \\
$A$ & $0.00545 \pm$ & $0.00013 \mathrm{~d}$ \\
$T_{0}$ & $0.0000 \pm$ & $0.0001 \mathrm{~d}$ \\
\hline
\end{tabular}

the presence of the third body (LITE) and the period $P_{2}=4465 \mathrm{~d}$ was preferred since it yielded a marginally better fit. The orbital elements are given in Table 2. Both programs were written by Dr. J. Horn at the Ondřejov Observatory and details of using these programs for analysis of LITE can be found in Šimon (1996). The value of eccentricity of the orbit of the possible distant companion is below the significance level given by the criterion of Lucy \& Sweeney (1971) and needs to be improved by the future observations.

The systematic shift of the fitting $T_{0}$ was interactively adjusted to zero. Although the full amplitude is just about $15 \mathrm{~min}$ also the averaged visual data generally follow the course of the photoelectric ones, as can be seen in Fig. 3.

The mass function of the third body is $f\left(m_{3}\right)=$ 0.006405 . The observed semi-axis of the eclipsing pair orbiting around the distant companion is $a_{12} \sin j=0.99 \mathrm{AU}$ where $j$ denotes an inclination angle of the orbit of the third body. The expected semi-amplitude $K(R V)$ of changes of the systemic velocity accompanying LITE is $2.5 \mathrm{~km} \mathrm{~s}^{-1}$ and this shift could be possibly detected in a set of high-dispersion spectra secured in the course of several years.

A set of the parametric solutions of the mass of the suspected third body in TT Aur is given in Table 3. The minimum mass of this distant companion is $1.11 M_{\odot}$ what corresponds to spectral type G3V. Its mass grows with decreasing angle $j$ and reaches $2.50 M_{\odot}$ for $j=28.1^{\circ}$ (B9.5V). A companion with such an early spectral type could be already revealed in the solution of the light curve as the third light. Nevertheless, such an analysis by Bell et al. (1987) did not reveal this excess light and we can therefore conclude that this companion, if present, is rather a low-mass star of medium or later spectral type.

The cycles with $P_{2}$ are plotted also for the earlier data in Fig. 3. The $\mathrm{O}-\mathrm{C}$ values of the old data which standard deviation is 0.005 days tend to be systematically more positive within $E=-24500$ to -14500 in comparison with the newer timings. Increase of the orbital period of the eclipsing pair can give a plausible explanation. The whole data set was fitted by a parabola and yielded $\Delta P / P \approx 1.210^{-10}$ days $^{-1}$. This parabolic increase has significance $S=1.37$ (after subtraction of the cyclic 


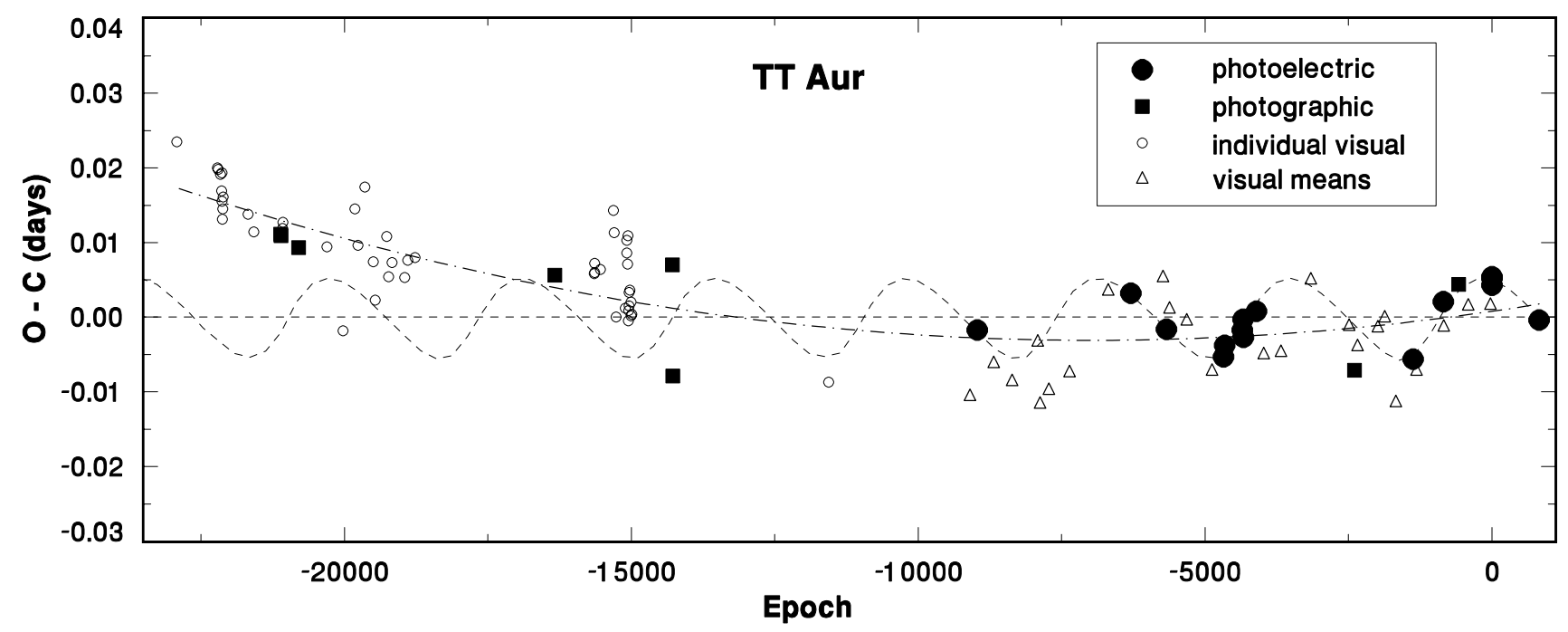

Fig. 3. The O-C curve of TT Aur calculated according to the ephemeris in Eq. (3). The period changes appear to be complex. The cyclic variations are well defined by the photoelectric timings (see also Fig. 4). The means of four visual timings were used for $E>-10000$ while the individual visual observations are plotted for the older data. The solution for LITE with the parameters given in Table 2 is repeated for the whole data set. The earliest data speak in favour of a long-term increase of the period. The dot-dashed line represents the parabolic fit to the whole data set. See the text for details

Table 3. The set of parametric solutions of the mass of the third body in TT Aur. The spectral type corresponds to the appropriate mass of a main sequence star given by Harmanec (1988). The visual absolute magnitudes $M_{V}$ were adapted from $M_{\mathrm{Bol}}$ in the same source and corrected for BC

\begin{tabular}{cccc}
\hline Mass $\left(M_{\odot}\right)$ & Inclination $j$ & Spectr. type & $M_{V}$ \\
\hline 2.50 & $28.1^{\circ}$ & B9.5 & 0.90 \\
2.00 & $35.3^{\circ}$ & A3 & 1.75 \\
1.50 & $48.9^{\circ}$ & F0 & 2.94 \\
1.11 & $89.1^{\circ}$ & G3 & 4.34 \\
\hline
\end{tabular}

variations). The $\mathrm{O}-\mathrm{C}$ variations in TT Aur can be thus described as a superposition of a parabolic trend and cyclic changes.

\subsection{BF Aur}

This system is exceptional in this ensemble since its mass ratio $q$ is very close to unity. Leung (1989) classified this binary as an inverse Algol $(q>1)$ where the more massive star is more advanced in its evolution and fills in its Roche lobe while the less massive component is still inside its lobe. Kallrath \& Kamper (1992) preferred a detached configuration but the more massive (but less luminous) star is still only by about $2 \%$ smaller than its lobe. Demircan et al. (1997) have recently argued that the fainter but hotter and less massive component fills its lobe; $q$ would be smaller than unity and BF Aur would not be inverse Algol. Djurasevic et al. (1997) showed that solution with $q>1$ is possible, too, and Demircan et al. (1997) admitted that it is not possible to resolve between these models

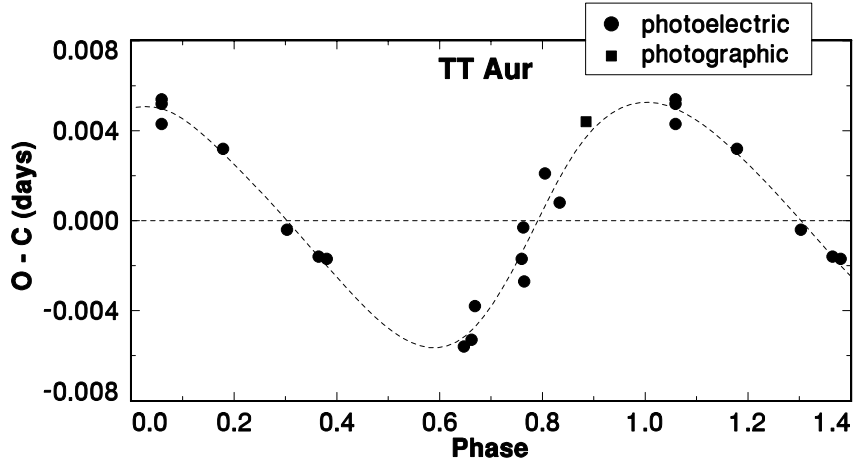

Fig. 4. The cyclic course of the $\mathrm{O}-\mathrm{C}$ values for TT Aur folded with the period of 4465 days. The smooth curve represents the orbital solution for LITE with the parameters given in Table 2

because they are based on the only one available set of radial velocity curves published by Mammano et al. (1974) which does not allow for exact determination of $q$. The solutions agree that both components are very similar to each other and also the differences in the parameters of both stars given by the respective authors are small. The parameters used in Table 1 come from Kallrath \& Kamper (1992).

The available timings cover about 95 years. Since the primary and secondary minima have almost identical light curves both were used for the construction of the $\mathrm{O}-\mathrm{C}$ diagram with times of the secondary minima shifted by $P / 2$. The elements with the orbital period given in Eq. (4) (taken from SAC96) and used in Fig. 5 satisfy the second half of the covered interval while the $\mathrm{O}-\mathrm{C}$ values in 


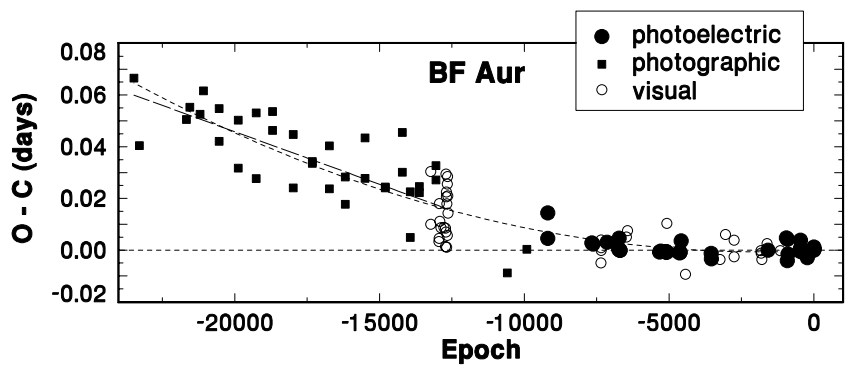

Fig. 5. The $\mathrm{O}-\mathrm{C}$ values for $\mathrm{BF}$ Aur covering about 95 years calculated according to the elements from SAC96. Both the primary and secondary minima (shifted by $P / 2$ ) were used. The fits of the data are displayed. See the text for details

the first half suggest a shorter period. Standard deviations of the photographic and visual timings are 0.009 and 0.005 days, respectively.

$T(\min \mathrm{I})=2449002.0255+1.58322190 \mathrm{E}$.

A change of the orbital period definitely occurred within the covered interval but since the photoelectric data are available only for about a half of the interval an exact determination of the character of this change is somewhat difficult. Both possibilities, parabolic course and constant period, are plotted for the interval of $E=-23000$ to -12000 in Fig. 5. Only photographic and visual timings are available there and their scatter is large therefore the resolving between parabolic course and constant period is impossible in this interval. The parabolic fit of the whole data set $(S=2.25)$ yields $\Delta P / P=$ $1.7510^{-10}$ days $^{-1}$ what is in a good agreement with the value $2.0710^{-10}$ days $^{-1}$ determined from a much shorter segment $(\Delta E \approx 9000)$ by Zhang et al. (1993). This fact speaks in favour of a rather continuous period change.

\subsection{AQ Cas}

This binary is a very massive system $\left(21.3 M_{\odot}\right)$ with long orbital period of 11.7 days. The available timings cover about 56 years. The elements given by Olson (1994) were used. They suit the data but the basic minimum should be 2426 282.43 JD rather than 2426282.34 JD. The corrected value is used in Fig. 6. Standard deviation of the visual and photographic data is 0.059 days. As $S=1.02$ suggests the period can be considered constant within the scatter of the data.

The mass transfer rate $\dot{m} \approx 510^{-7} M_{\odot} \mathrm{yr}^{-1}$ was determined from the photometric manifestations of the mass stream by Olson \& Bell (1989). In order to assess how large $\dot{m}$ for the conservative transfer (Huang 1963) can be hidden in the scatter of the data parabolic courses for a set of $\dot{m}$ were computed and are included in Fig. 6. It can be seen that the expected course of the period change for $\dot{m}$ determined by Olson \& Bell (1989) is too small to be unambiguously present in the available data.

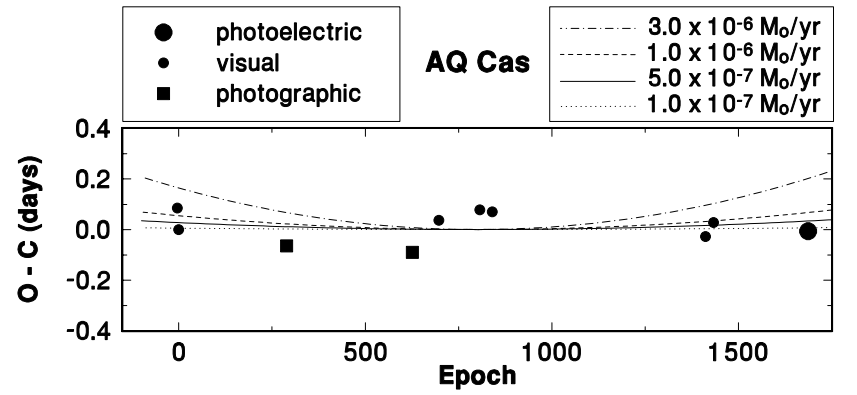

Fig. 6. The $\mathrm{O}-\mathrm{C}$ curve for $\mathrm{AQ}$ Cas. The data are consistent with the constant period during the whole interval. A set of parabolas represents the expected period changes assuming the conservative case and various values of $\dot{m}$. See the text for details

It is interesting to note that although the parameters and position of AQ Cas in the $r-q$ and $P-q$ diagrams in Fig. 17 are very similar to $\beta$ Lyr (see below) the magnitudes of the period changes are very different.

\section{6. $X Z C e p$}

The mass transfer in XZ Cep is still proceeding since the spectral lines, namely those of the Balmer series, are contaminated by CM (Glazunova \& Karetnikov 1985).

The orbital period of XZ Cep is variable (Kreiner et al. 1990). These authors offered a parabolic fit of the O-C values. The timings contained in the Lichtenknecker database are the same as those analysed by Kreiner et al. (1990). The $\mathrm{O}-\mathrm{C}$ values calculated according to their Eq. (3) (Fig. 7) were reexamined. The covered interval is about 57 years long and an increase of the orbital period is evident $(S=2.21)$. Nevertheless, the character of this change is not quite clear. The $\mathrm{O}-\mathrm{C}$ values within $E=2300$ to 4100 , i.e. 25 years, are consistent with the constant period. A group of timings within $E=0$ to 400 has significantly more positive $\mathrm{O}-\mathrm{C}$ values. Standard deviation of these mostly visual data is 0.016 days. There also exists an alternative to the parabolic trend: a possible abrupt change which took place at $E=2300$ or sooner. In this case a lower limit of the period change is $\Delta P / P \approx 0.7210^{-5}$ days $^{-1}$. Both alternatives, parabolic fit and a lower limit of the abrupt change, are shown in Fig. 7 and as can be seen they are indistinguishable at present.

\subsection{448 Cyg}

$\mathrm{V} 448 \mathrm{Cyg}$ is a system with the total mass about $39 M_{\odot}$ and the less massive secondary star fills in its lobe (Harries et al. 1997). The mass transfer is proceeding as was documented by an analysis of the emission in $\mathrm{H} \alpha$ by Volkova et al. (1993). This emission was interpreted in terms of two components: (1) the mass outflowing through the $L_{2}$ point; (2) the mass streaming from 


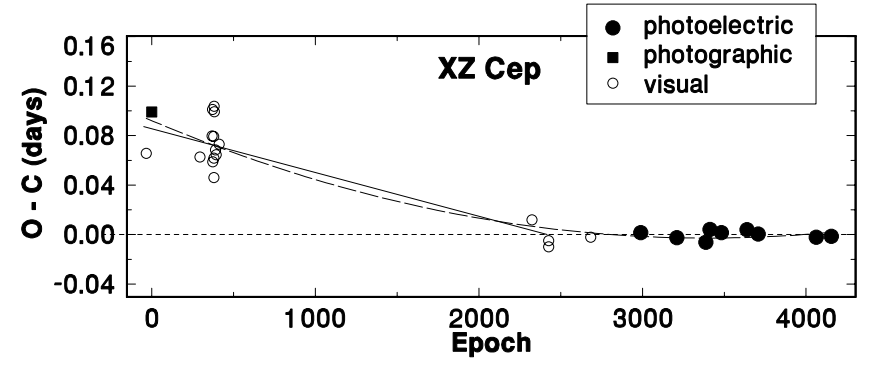

Fig. 7. The O-C curve for XZ Cep. The data within $E=2300$ to 4100 are consistent with a constant period while the old data suggest increase of the period somewhere inside the covered interval. Both parabolic trend and lower limit of an abrupt change are displayed. See the text for details

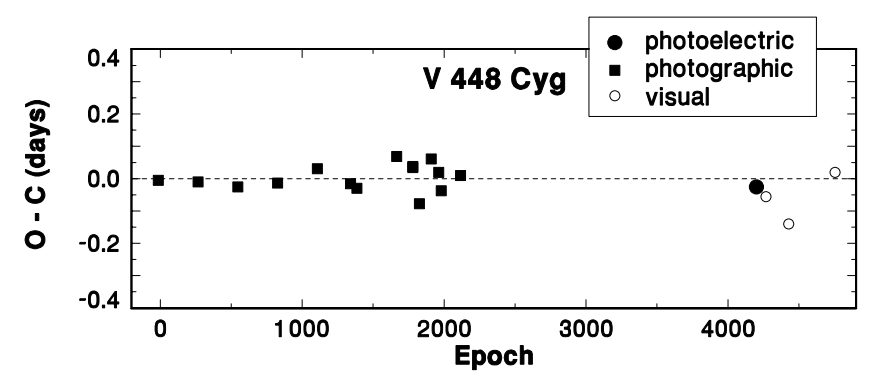

Fig. 8. The O-C curve for V 448 Cyg. The whole data set is consistent with the constant period

the loser towards the gainer. The light curve is variable and the changes are prominent namely in the secondary minimum (Zakirov 1992).

The $\mathrm{O}-\mathrm{C}$ values calculated according to the elements from SAC96 (Eq. (5)) are displayed in Fig. 8. Standard deviation of the photographic and visual data is 0.047 days. The total length of the covered interval is about 86 years and although there is a gap in the data the course of the $\mathrm{O}-\mathrm{C}$ values is consistent with the constant period within the accuracy of the timings $(S=1.11)$.

$T(\min \mathrm{I})=2416361.107+6.5197162 \mathrm{E}$.

\section{8. u Her}

The system is semi-detached with the primary being the main sequence star while the evolved secondary is relatively larger and overluminous for its mass (Koch et al. 1970; Eaton 1978). A difference in RVs of the triplets and singlets of HeI amounting $13 \mathrm{~km} \mathrm{~s}^{-1}$ was interpreted in terms of contamination by circumstellar material (Hilditch $\&$ Hill 1975). The gas streams were inferred from variations in the equivalent heights of the absorption lines of the primary by Kovachev \& Reinhardt (1975).

A very extensive set of timings is available for this bright binary. Nevertheless, as a detailed examination showed the visual data display appreciable scatter. Since

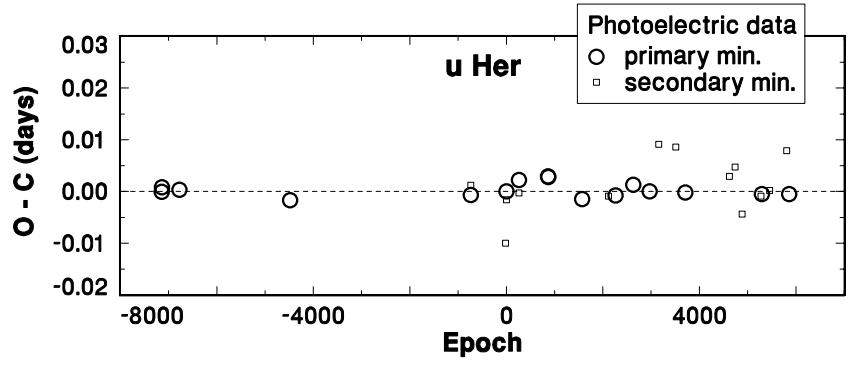

Fig. 9. The $\mathrm{O}-\mathrm{C}$ values for $\mathrm{u}$ Her. Only the photoelectric data covering the interval 73 years long were used. The elements in Eq. (6) were used and it can be seen that the period was constant in the whole interval

the photoelectric timings cover an interval about 73 years long it was decided to base the analysis just on these data. The period given in SAC96 was found to be too long. Its new value was determined from the moments of the primary minima in the whole covered interval and the corrected elements can be found in Eq. (6).

$T(\min \mathrm{I})=2436831.3040+2.051026168 \mathrm{E}$.

The $\mathrm{O}-\mathrm{C}$ values for the primary minima (Fig. 9) are fully consistent with the constant orbital period inside the whole interval $(S=1.07)$ and confirm the finding by Kreiner \& Ziolkowski (1978). The secondary minima, shifted by $P / 2$, are displayed, too. As can be seen they are scattered more than the primary minima and may display a marginal tendency to occur later by up to 0.01 days.

\subsection{VYLac}

VY Lac is a very close binary (see Fig. 17) consisting of two early-type components but the configuration of this system is based just on the light curve solution (Semeniuk \& Kaluzny 1984) and no RV curves are available. The absolute masses and radii presented by these authors and listed in Table 1 are based on the statistical mass and radius of the primary component.

$T(\min \mathrm{I})=2448180.3474+1.036243933 \mathrm{E}$.

The orbital period given in SAC96 is too short and does not satisfy the whole data set. New elements based only on the photoelectric timings were determined and are given in Eq. (7). The total covered interval is 66 years long and it can be seen in Fig. 10 that also the older photographic and visual minima are in accordance with the elements in Eq. (7). Standard deviation of the visual and photographic timings is 0.004 days. We can conclude that the data suggest constant period in the last 66 years $(S=1.00)$.

\subsection{0. $\beta$ Lyr}

$\beta$ Lyr is a well-known long-period (13 days) system which displays an exceptionally strong activity among the binaries which contain only non-degenerate stars. Reviews 
10

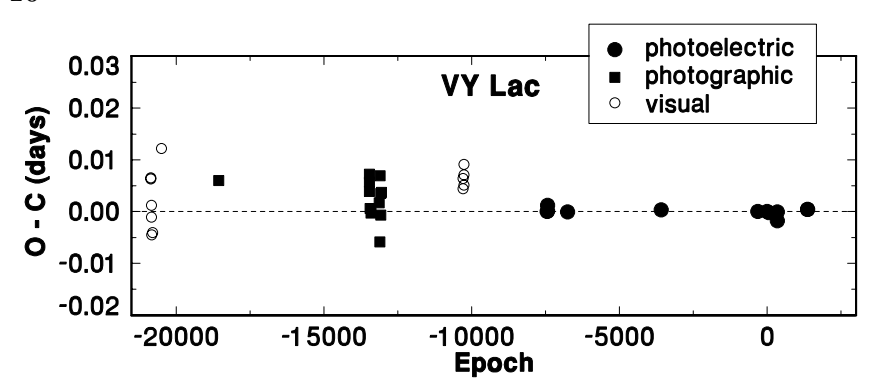

Fig. 10. The O-C curve for VY Lac. The ephemeris in Eq. (7) well satisfies the data and the period can be considered constant within the whole interval of 66 years

of the research of this system can be found in Sahade \& Wood (1978) and Harmanec et al. (1996). Let us only note that $\beta$ Lyr displays strong emission lines in its spectrum and the large underluminosity of the gainer was interpreted in terms of a huge opaque accretion disk (Wilson 1974). The orbital period of $\beta \mathrm{Lyr}$ is increasing at a high rate with $\Delta P / P=5.99710^{-7}$ days $^{-1}$ and the course of the $\mathrm{O}-\mathrm{C}$ changes can be well approximated by a parabola, giving the mass transfer rate of the order of $210^{-5} M_{\odot} \mathrm{yr}^{-1}$ from the less massive loser onto the more massive gainer (Harmanec \& Scholz 1993). They argued that the transfer in case of $\beta$ Lyr can be regarded as approximately conservative. Moreover, Harmanec and Scholz found that also an increase of the semi-amplitude of radial velocity variations of the loser as a response to its mass loss is possible.

The large optically thick disk embedding the gainer suggests large mass inflow. Although the value of the mass accretion rate onto the gainer inferred from the model of this disk by Hubený \& Plavec (1991) is about five times larger than $\dot{m}$ determined from the $\mathrm{O}-\mathrm{C}$ change by Harmanec and Scholz the agreement is not bad and confirms exceptionally large $\dot{m}$. Some departure from a purely conservative mode was admitted and jets of the outflowing matter were later used as an interpretation of the spectroscopic and interferometric observations by Harmanec et al. (1996).

\subsection{DM Per}

The elements for this system published in SAC96 were slightly corrected and the revised ephemeris given in Eq. (8) was used for calculation of the O-C values (Fig. 8). Only the photoelectric and two visual timings were used since the rest observations displayed a large scatter. The data are consistent with the constant period inside the covered interval $(S=1.08)$.

$T(\min \mathrm{I})=2444855.5057+2.727741844 E$.

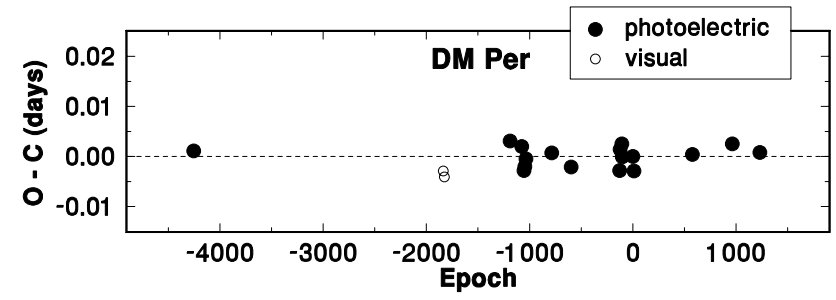

Fig. 11. The $\mathrm{O}-\mathrm{C}$ values for $\mathrm{DM}$ Per. The data are consistent with the constant period in the whole interval of the observations

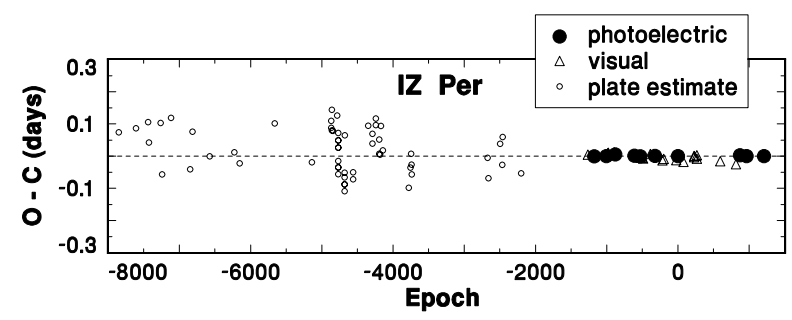

Fig. 12. The O-C curve for IZ Per. The whole data set is consistent with the constant orbital period

\subsection{IZ Per}

Only relative dimensions of this semi-detached system, listed in Table 1, are available. They were determined from the solution of the light curve by Wolf \& West (1993). The absolute radii and masses could not be determined since the only one RV curve of the primary, published by Yavuz (1969), leads to an unacceptably low mass of this star (only $2.4 M_{\odot}$ ), much smaller than corresponds to its spectral type B3-4 determined by Wolf \& West (1993) from its spectrum. Since both components are of early spectral types the RV curve affected by a line blending can be a plausible explanation for this discrepancy.

The ephemeris given in SAC96 (Eq. (9)) fits the photoelectric and visual timings within $E=-1300$ to 1100 (24 years) very well and the period is constant there with $S=1.05$ (Fig. 12). Standard deviation of the visual data is 0.010 days. Only estimates from the archival photographic plates are available for the earlier timings and their scatter is large ( $\sigma=0.067$ days). Nevertheless, also these data are consistent with the constant period during the last 92 years.

$T(\min \mathrm{I})=2444577.5874+3.687673 \mathrm{E}$.

\subsection{3. $R Y S c t$}

The parameters and namely the configuration of this enigmatic system are still a matter of debates. The results agree on the facts that the total mass of RY Sct is exceptionally high and that both stars are of early spectral types. The system is certainly strongly interacting and a 


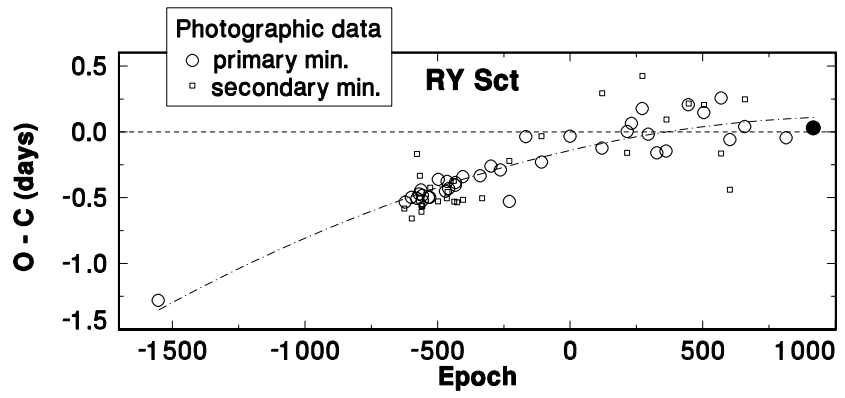

Fig. 13. The course of the $\mathrm{O}-\mathrm{C}$ values for $\mathrm{RY}$ Sct calculated according to Eq. (10) (SAC96). Fit of the primary minima by the second-order polynomial is displayed, too

large mass outflow even led to formation of an extended asymmetric envelope (e.g. de Martino et al. 1992).

An overcontact configuration emerged from the solution of the light curve (Milano et al. 1981). On the contrary, King \& Jameson (1979) preferred a semi-detached model of the $\beta$ Lyr-type in their discussion of the spectroscopic observations. The recent analysis of the RV curves of both components by Skulskij (1992) led to a quite new value of the mass ratio $q=0.301$ and smaller masses of both stars than was supposed previously. His model suggests a semi-detached configuration with a huge accretion disk around the detached gainer and is similar to the model by King and Jameson. In this context it is useful to note that as was demonstrated by the models of Zola (1995) an optically thick accretion disk completely veiling the gainer in a semi-detached binary can seriously alter the results of the light curve solution and the system can even pretend a contact configuration. The contact configuration of RY Sct obtained from photometry by Milano et al. (1981) and the semi-detached model of Skulskij (1992) and King \& Jameson (1979) could be reconciled in this way. In any case, new solution of the light curve of RY Sct using Zola's approach is necessary. We will therefore make use of the model by Skulskij (1992) in the following analysis.

The previous analysis of the period changes by Milano et al. (1981) revealed that the period of RY Sct decreases. An interpretation in terms of the mass loss or transfer was offered.

The $\mathrm{O}-\mathrm{C}$ values for the data available at present were calculated according to the elements from SAC96 (Eq. (10)) and are plotted in Fig. 13. Almost all timings were obtained from the photographic observations of minima (not plate estimates). Since the depth of the primary and secondary minimum is comparable timings of both are available. They are marked by different symbols in Fig. 13 . The course of the $\mathrm{O}-\mathrm{C}$ changes for the primary minima is somewhat better defined (standard deviation of the photographic timings 0.078 days) but it is not possible to say now if this is caused purely by observational inaccuracies or if real changes of the light curve play a role, too. The full length of the data set confirms the decrease of the period $(S=1.79)$. The O-C values for the primary minima were fitted by the second-order polynomial, displayed in Fig. 13. The corresponding rate of the period change is $\Delta P / P=-3.7110^{-8}$ days $^{-1}$. Owing to the scatter of the data determination of any more detailed course is not meaningful at present.

$T(\min \mathrm{I})=2432796.477+11.124138 \mathrm{E}$.

As King \& Jameson (1979) and Skulskij (1992) argued the less massive star fills in its Roche lobe and transfers mass onto its more massive companion. The course of the observed period change, that is its decrease, is in the opposite sense than would correspond to the dominant conservative mode (see also below).

\subsection{RZ Sct}

This binary is a long-period (15.2 days) system consisting of two evolved stars (Olson \& Etzel 1994, hereafter OE94). The large radius of the evolved gainer is the reason why this binary is placed well inside the area of direct impactors in the $r-q$ diagram despite of its long orbital period (Fig. 17, see also below). The activity connected with the mass transfer is well documented for this system: the RV curve is distorted (e.g. McNamara 1957), emission in $\mathrm{H} \alpha$ is visible outside eclipses (McNamara 1957; Hansen \& McNamara 1959), the light curve displays distorsions by the stream (Olson \& Bell 1989).

The temperature of the loser is somewhat uncertain. The values given by various authors differ but agree on spectral type A. Recent solution of the light curve by OE94 yields $T_{2}=6418 \mathrm{~K}$. This value leads to spectral type later (about mid-F) than given in previous analyses and may shift the loser into area of stars with COL. Nevertheless, it was decided to retain RZ Sct in the ensemble, namely because of the value of $\dot{m}$ available for this system (see below).

The available timings cover about 77 years and the $\mathrm{O}-\mathrm{C}$ values were calculated using the ephemeris given by OE94 in the first step. These authors determined the period from their photoelectric observations done in 1983 - 1990 and although their minima have not been published this period is definitely too long and yields $\mathrm{O}-\mathrm{C} \approx+1.6$ days for the earliest timings. After several trials it was found that the ephemeris given in Eq. (11) satisfies most of the data (Fig. 14).

$T(\min \mathrm{I})=2436942.560+15.19024195 \mathrm{E}$.

We can see that a large part of the $\mathrm{O}-\mathrm{C}$ values $(E=$ -1200 to 0 ) is consistent with the constant period and the scatter is formed by timings of a lower weight (standard deviation of the visual data 0.058 days). There is a possible lengthening of the period after $E=0$. Five visual minima after $E=0$, although affected by a scatter, have more positive $\mathrm{O}-\mathrm{C}$ values than the earlier timings. Two photoelectric minima can be resolved around $E=0$; the latter 


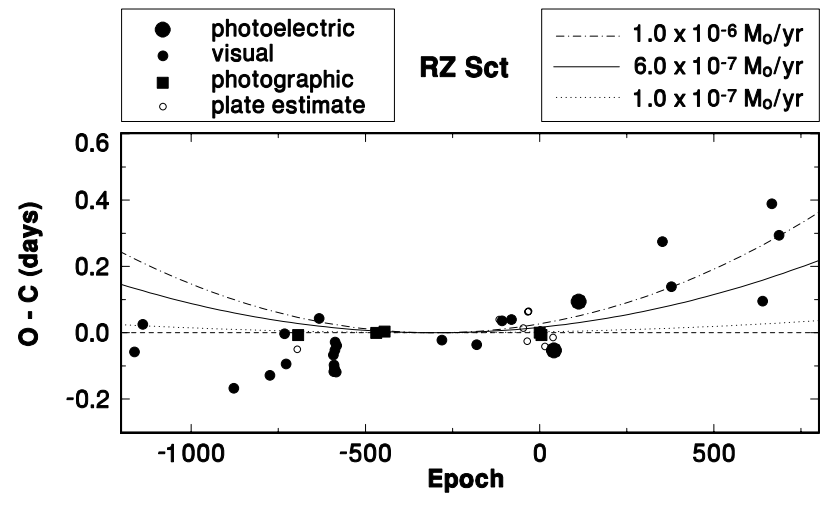

Fig. 14. The $\mathrm{O}-\mathrm{C}$ curve for RZ Sct. Although the high mass transfer rate $\dot{m} \approx 610^{-7} M_{\odot} \mathrm{yr}^{-1}$ was determined by Olson \& Bell (1989) the period change is very small (if any). A set of parabolas represents the expected period changes assuming conservative case and various values of $\dot{m}$. See the text for details

has a more positive $\mathrm{O}-\mathrm{C}$ value. This increase of the period length is also in accordance with the longer $P$ found by OE94 for the interval of years 1983 - 1990. The difference between the period given by OE94 and that in Eq. (11) is $\Delta P / P \approx 710^{-5}$ days $^{-1}$. The linear fit to the minima after $E=0$ yields a smaller period change with respect to the period length in Eq. (11) about $\triangle P / P \approx 310^{-5}$ days $^{-1}$. Significance $S$ of the period change is not very low ( $S=1.49$ ) but since this change is dependent to a large extent on the scattered visual data taken after $E=0$ it must be regarded as uncertain.

The sense of this possible period change is in accordance with the expected period variation in the dominant conservative mass transfer in a binary where the loser is less massive than the gainer. Olson \& Bell (1989) determined the mass transfer rate $\dot{m} \approx$ $610^{-7} M_{\odot} \mathrm{yr}^{-1}$. Various manifestations of continuing mass transfer through the years cited above are available. Moreover, as can be inferred from the photometry of OE94 the shape of the light curve with the distorsion caused by the mass stream was quite stable for at least seven years. It is not therefore unreasonable to suggest that the value of $\dot{m}$ determined by Olson \& Bell (1989) is typical for RZ Sct in the interval covered by the timings. The appropriate change of period expected for the conservative case can be then compared to the observations in the same way as for AQ Cas. The data do not contradict the increase of $P$ which corresponds to $\dot{m}$ of Olson \& Bell (1989).

\subsection{5. $V 356 \mathrm{Sgr}$}

This is a massive system $\left(16.8 M_{\odot}\right)$ with the orbital period 8.9 days long. The B3 primary is supposed to be critically rotating and surrounded by a geometrically thick, opaque and non-luminous disk (Wilson \& Caldwell 1978).
Strong UV emission lines are visible during the total primary eclipse (Plavec et al. 1984). The photometric and spectroscopic observations in the UV band obtained with IUE revealed variable eclipse light curve and non-uniform distribution of the circumstellar matter, located namely out of the orbital plane (Polidan 1989). An outflow of mass from the system was suggested.

The timings in the Lichtenknecker database contain only the data already published by Wilson \& Woodward (1995). Although the number of timings is small Wilson and Woodward argued that they did not give any evidence of a significant period change and might be even consistent with a constant period, in contradiction with the evolutionary computations by Ziolkowski (1985).

\subsection{Z Vul}

A very extensive set of timings obtained by various methods is available for this binary. The visual timings which are the most numerous were grouped into bins of ten observations. The plot of the $\mathrm{O}-\mathrm{C}$ values calculated according to the elements from SAC96 revealed clearly variable period $(S=4.84)$. Standard deviations of the individual photographic timings and means of ten visual data are 0.004 and 0.0027 days, respectively. New ephemeris which keeps a low slope of a large part of the $\mathrm{O}-\mathrm{C}$ values and thus shows the course with the best clarity was determined from the initial elements and is given in Eq. (12). The corresponding plot can be seen in Fig. 15. Although the period length definitely increased inside the covered interval it is difficult to resolve the true character of this change. Namely the photoelectric data bring some evidence for a possible episode of an abrupt change around $E=1100$. Both possibilities are shown in Fig. 15. The parabolic course yields $\Delta P / P=2.8710^{-10}$ days $^{-1}$ while the magnitude of the eventual abrupt change would be $\Delta P / P=2.310^{-6}$ days $^{-1}$.

$T(\min \mathrm{I})=2435381.3844+2.454926352 \mathrm{E}$.

The photoelectric timings in the segment marked by shorter arrowed line (after the possible episode) in Fig. 15 were used for determination of the elements which are given in Eq. (13). This ephemeris served for calculation of the $\mathrm{O}-\mathrm{C}$ values which are displayed in Fig. 16.

$T(\min \mathrm{I})=2447744.4145+2.454932059 \mathrm{E}$.

\section{Comprehensive characteristics of the systems}

Since the physical parameters of the binaries listed in Table 1 are known the present analysis of the orbital period changes allows for a search for possible general relations between the system parameters and the period changes. 


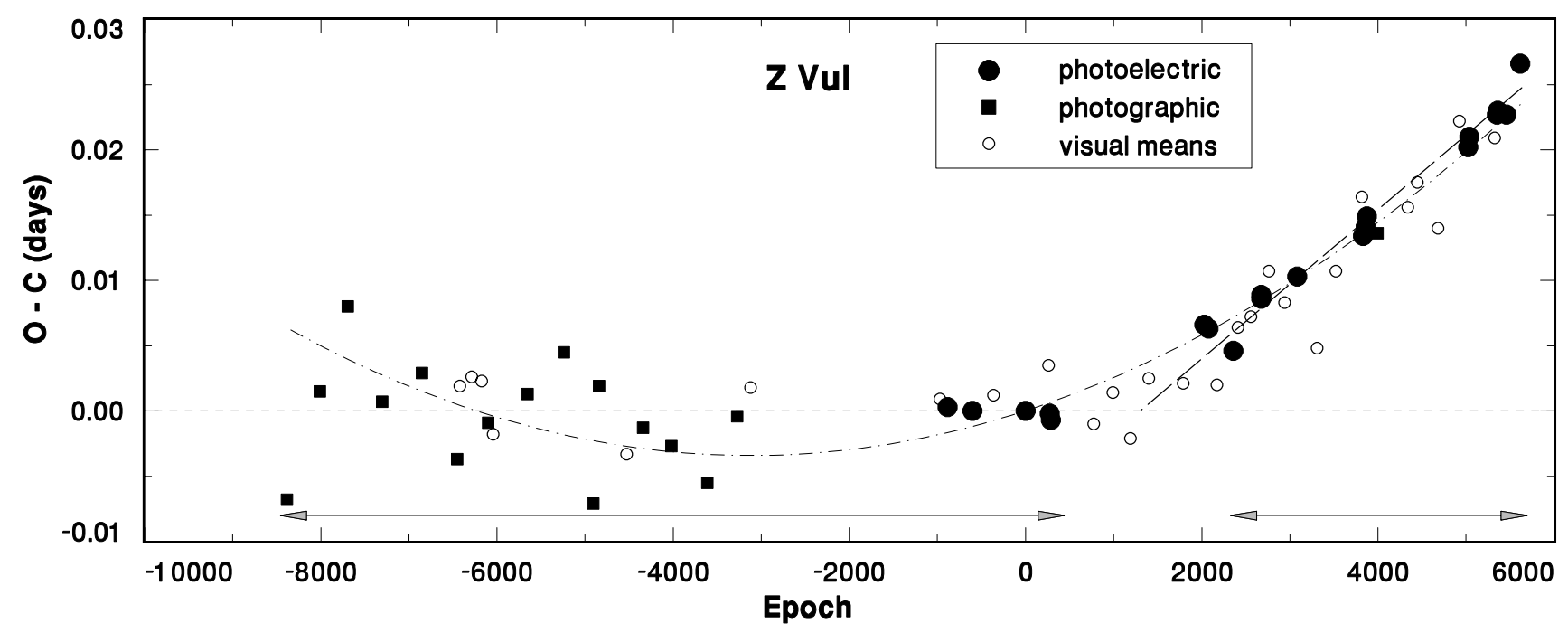

Fig. 15. The plot of the $\mathrm{O}-\mathrm{C}$ values of $\mathrm{Z}$ Vul calculated using Eq. (12). The segment marked by the longer arrowed line was used for determination of this ephemeris. Both parabolic fit and a possible abrupt change of period are shown. See the text for details

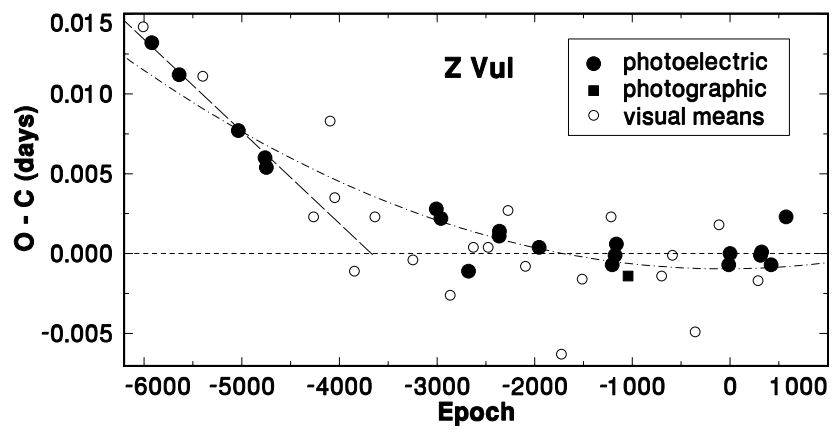

Fig. 16. Segment of the data from Fig. 15 showing the detail of the possible episode of abrupt period change in $\mathrm{Z} \mathrm{Vul.} \mathrm{The}$ $\mathrm{O}-\mathrm{C}$ values were calculated using Eq. (13). The long-dashed line represents the course given by Eq. (12). The parabolic fit from Fig. 15 is plotted, too. See the text for details

The so called $r-q$ diagram, although used just for assessment of the properties of the accretion disks (e.g. Peters 1989), plays an even more important role since it contains information not only about the fate of the transferred matter, but also about the evolutionary status of the binary, tidal effects and the influence of the mass stream on the gainer. This diagram displays $r=R_{\mathrm{g}} / a$, which is the radius of the gainer $R_{\mathrm{g}}$ expressed in terms of fraction of the orbital separation $a$, versus the mass ratio $q$. Position of a system in this diagram allows to determine the fate of the stream of the transferred matter in the ballistic approximation. As Lubow \& Shu (1975) calculated two curves can be plotted. The curve $\omega_{\text {min }}$ denotes the minimum distance of the infalling stream from the center of the gainer. A permanent disk can develop in systems lying below this curve because the stream completely misses the gainer. The curve $\omega_{\mathrm{d}}$ gives the radius of the disk which is formed from the infalling matter. Systems lying between $\omega_{\min }$ and $\omega_{\mathrm{d}}$ can have only transient disks which are mixture of the matter of the stream and the photosphere splashed by impact of the infalling stream. On the other hand, the impact of the stream onto the gainer is almost tangential to its photosphere in systems with large $r$. Generally, the higher above the curve $\omega_{\min }$ the system lies, the more difficult conditions for development of the disk. Also the interaction of the gainer with the stream becomes stronger in systems with larger $r$.

All systems analysed here are plotted in the $r-q$ diagram in Fig. 17a. Different symbols were used to resolve the systems with variable periods from those which periods can be regarded as constant during the whole interval of observations. It can be seen that all systems lie above the curve $\omega_{\text {min }}$, i.e. the stream collides at least partly with the gainer. Moreover, most of these binaries (thirteen of sixteen) are situated inside the area of direct impactors above $\omega_{\mathrm{d}}$ and some even have gainer almost filling its lobe. These facts suggest that the tidal interaction is appreciable in most binaries of this set and also the gainers are strongly affected by the impact of the transferred matter.

Similar diagram can be seen in Fig. 17b where the mass ratio is plotted versus the orbital period. The same symbols as in Fig. 17a were used. Most systems with variable periods occupy the upper-left corner of the $P$ - $q$ diagram. This fact may be to some extent caused by the selection effect since the binaries with longer periods have larger orbital separation and the probability of observing deep eclipses is generally lower. Notice that there is a well visible tendency for the large mass ratios to occur in systems with the shortest periods.

The absolute value of the rate of the period change $\Delta P / P$ plotted versus the mass ratio $q$ can be seen in 

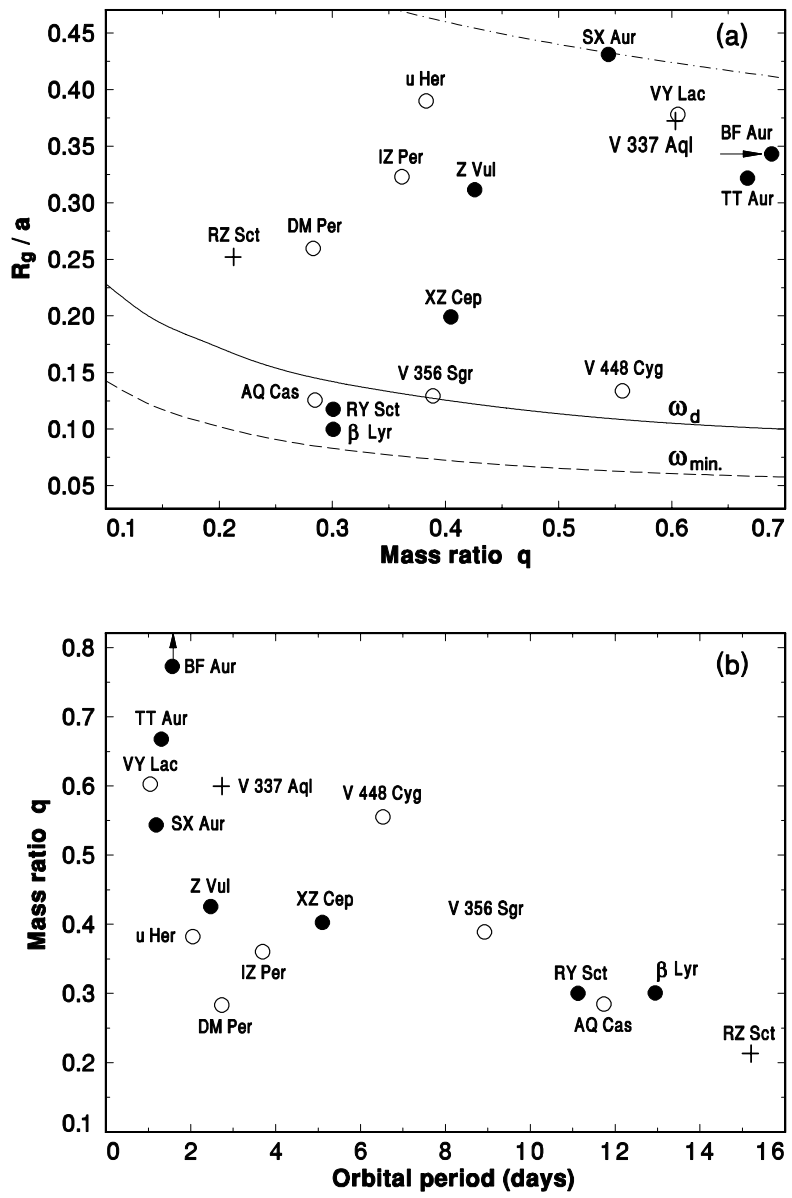

Fig. 17. The positions of the analysed binaries in the $r-q$ diagram a) and in the $P-q$ diagram b). The dot-dashed line marks the equivalent radius of the Roche lobe as a fraction of the orbital separation in a) and the meaning of the curves $\omega_{\min }$ and $\omega_{\mathrm{d}}$ is explained in the text. Systems with variable periods are marked by filled circles while empty circles denote binaries where no period change was observed during the whole interval covered by the data. Unsure cases are marked by crosses. See the text for details

Fig. 18. Only those systems where the stability of the period or its change could be determined with a good degree of accuracy were plotted. Some trends can be distinguished here with the help of the $r-q$ and $P-q$ diagrams: (a) most systems (six of eight) with $P<7$ days $\left(R_{\mathrm{g}} / a>\omega_{\mathrm{d}}\right)$ and with $q>0.4$ display variable period within $|\Delta P / P|=1.210^{-10}$ days $^{-1}$ to $3.2210^{-9}$ days $^{-1}$; (b) periods of binaries with $P<7$ days $\left(R_{\mathrm{g}} / a>\omega_{\mathrm{d}}\right)$ but with $q<0.4$ can be considered constant on the time scale of several decades; (c) two systems ( $\beta$ Lyr and RY Sct) with $\omega_{\min }<R_{\mathrm{g}} / a<\omega_{\mathrm{d}}(P>11$ days $)$ and with $q<0.4$ display extremely large period changes; although the parameters of AQ Cas are similar to these two systems the absence of period change moves it to group (b). We admit that the number of binaries used is not high but as we noted above the limitation is given by the requirement of

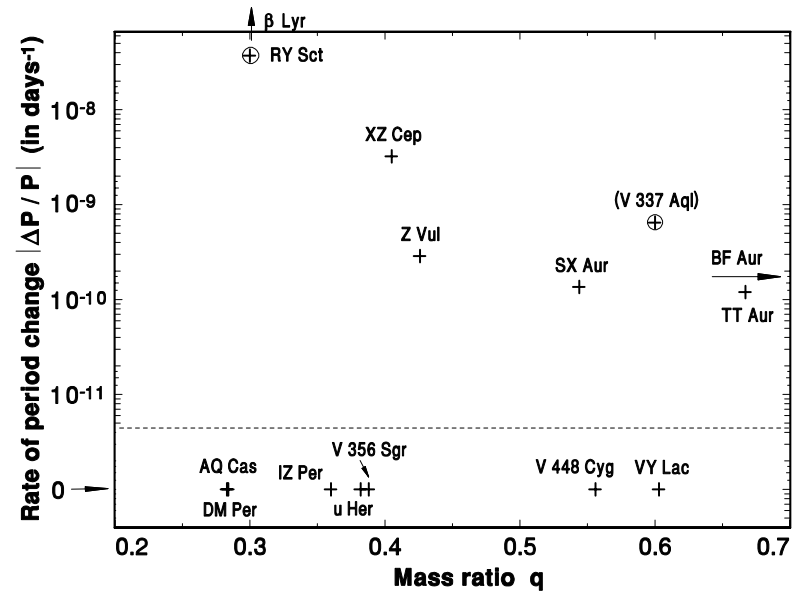

Fig. 18. Dependence of the rate of the period change $\Delta P / P$ (in days ${ }^{-1}$ ) on the mass ratio $q$. Absolute value of the period change was used and the systems which have $\Delta P / P<0$ are marked by circled crosses. See the text for details

both a good model and sufficient coverage by the timings for each system.

The loss of matter from the loser leads to considerable changes of its parameters through the epoch of the mass transfer. The status of the loser and the degree of progress of the mass exchange can be illustrated by the position of this star in the mass-luminosity diagram (Fig. 19). The mass of the loser decreases in the course of the epoch of the outflow of matter. The star therefore moves to the left (towards smaller masses) in this diagram. The luminosity initially decreases in the phase of the rapid mass transfer but begins to grow again as the system enters the more advanced evolutionary stadium in which the mass transfer rate slows down. Luminosity of each loser was calculated from its radius and $T_{\text {eff }}$ given in Table 1 . In the case of IZ Per the parameters of the loser could be determined only from a combination of the statistical mass of the primary for its spectral type (taken from Harmanec 1988) and the light curve solution by Wolf \& West (1993). The mass and luminosity of the loser in IZ Per must be therefore taken with caution but even an error of $50 \%$ would not significantly alter the position of this star in Fig. 19.

\section{Rotational velocities of the gainers}

The stars in non-evolved detached close binaries with small eccentricities usually rotate synchronously. However, the situation is usually different in the semi-detached systems because the rotational velocity of the gainer may be significantly higher than the synchronous one (Stothers 1973).

The observed values of the rotational velocities of the gainers $V_{\mathrm{e}}$ are available only for eight systems of the ensemble analysed here. They are summarized in Table 4 


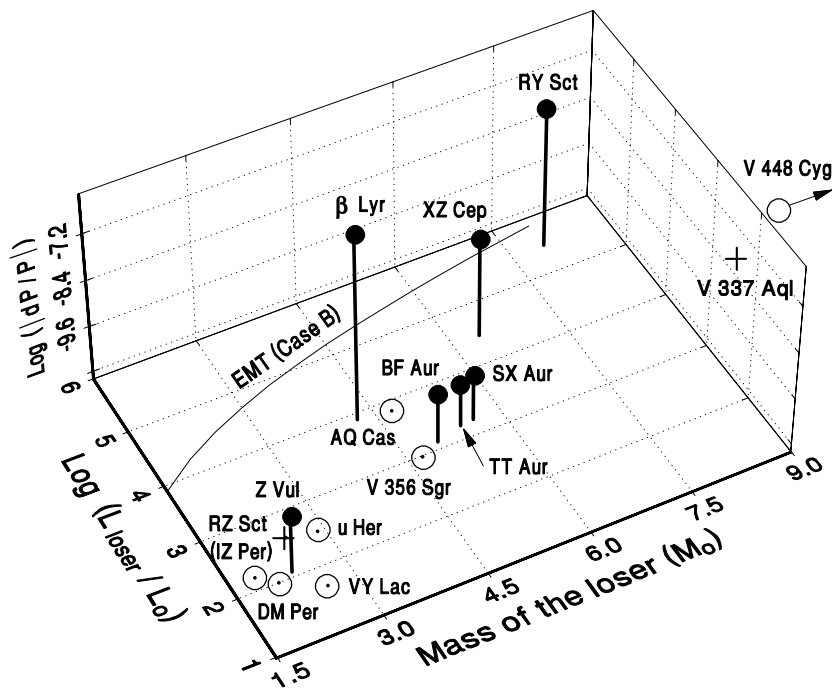

Fig. 19. Positions of the losers in the mass-luminosity diagram. The systems with constant periods are plotted as open circles lying in the $x y$ plane. Filled circles denote the binaries with variable periods and are shifted from the $x y$ plane by the distance which corresponds to the logarithm of absolute value of $\Delta P / P\left(\right.$ in days $\left.^{-1}\right)$, plotted on the vertical $(z)$ axis. Notice the prevailing location of the binaries with constant periods in the lower-left corner. The curve labeled as EMT marks the end of mass transfer for case B given by De Greve (1993)

along with the references. This table also contains the ratio of the real equatorial velocity to the synchronous one $V_{\mathrm{e}} / V_{\text {syn }}$. The synchronous value $V_{\text {syn }}$ was determined for the parameters of the system given in Table 1. Only in case of IZ Per the value of $V_{\mathrm{e}} / V_{\mathrm{syn}}$ was taken from the solution of the light curve by Wolf \& West (1993). Since it is not clear how $V_{\mathrm{e}}$ in V 448 Cyg listed by Volkova et al. (1993) was obtained this value should be taken with caution.

Dependence of the degree of asynchronous rotation of the gainer on $R_{\mathrm{g}} / a$ for these binaries is plotted in Fig. 20 . It can be immediately seen that systems which periods can be considered constant (AQ Cas, V 448 Cyg, IZ Per, V 356 Sgr) or where the change is uncertain (RZ Sct) have gainers rotating significantly faster than the synchronous value (often more than three times). The exception is $u$ Her. The gainers in two systems with variable periods, SX Aur and $\mathrm{Z} \mathrm{Vul}$, rotate rather close to the synchronous value. Certainly, the number of the systems is too small to confirm any clear relation of the rotational velocity of the gainer and the observed period changes but it is useful to evaluate the role of rotation of the gainer in the storage of angular momentum of the system (see below).

\section{Discussion}

A detailed analysis of the $\mathrm{O}-\mathrm{C}$ diagrams of ensemble of semi-detached binaries which contain only stars with radiative outer layers was performed. The summary of re-
Table 4. Rotational velocities of gainers in the semi-detached ET-systems. $V_{\text {e }}$ refers to the observed velocity corrected for the inclination angle while the synchronous value determined for the parameters given in Table 1 is abbreviated as $V_{\text {syn }}$. The ratio of the real to the synchronous rotation $V_{\mathrm{e}} / V_{\mathrm{syn}}$ is given, too. The last column gives the source of $V_{\mathrm{e}}$

\begin{tabular}{lrrll}
\hline Binary & $V_{\mathrm{e}}$ & $V_{\text {syn }}$ & $V_{\mathrm{e}} / V_{\text {syn }}$ & Ref. \\
\hline SX Aur & 243 & 217 & 1.1 & 1 \\
AQ Cas & 240 & 33 & 7.3 & 2 \\
V 448 Cyg & 100 & 52 & 1.9 & 5 \\
u Her & 119 & 143 & 0.83 & 1 \\
IZ Per & 185 & & 3 & 3,4 \\
RZ Sct & 217 & 53 & 4.1 & 3 \\
V 356 Sgr & 213 & 34 & 6.3 & 3 \\
Z Vul & 135 & 97 & 1.4 & 3 \\
\hline
\end{tabular}

References: 1. Stothers (1973); 2. Etzel \& Olson (1993); 3. Van Hamme \& Wilson (1990); 4. Wolf and West (1993); 5. Volkova et al. (1993).

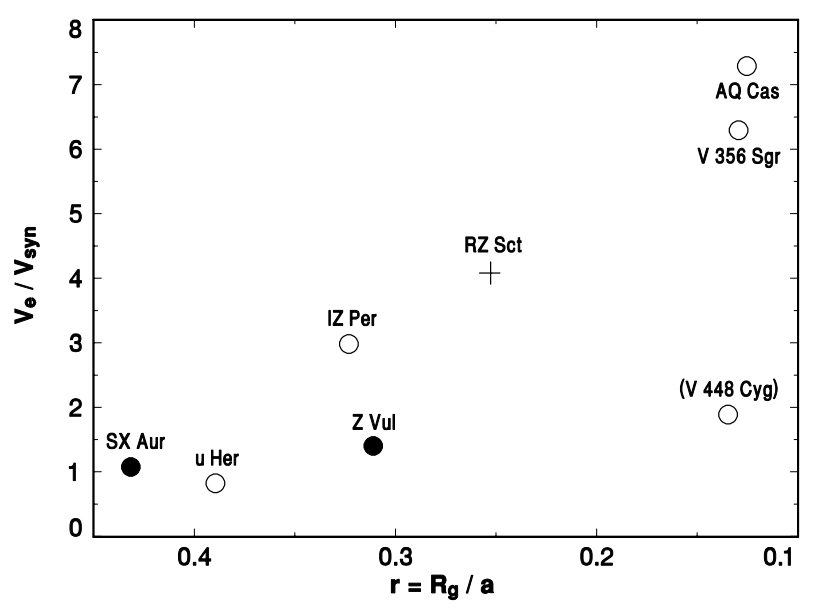

Fig. 20. Dependence of the degree of asynchronous rotation of the gainer on $R_{\mathrm{g}} / a$. The symbols are the same as in Fig. 17 . See the text for details

sults can be found in Table 5. The periods are constant, or display only monotonic changes, in the intervals covered by the timings. These intervals are several decades long (even more than 70 years in most cases). With one exception no signs of complicated course were detected in the $\mathrm{O}-\mathrm{C}$ diagrams of the respective systems within the covered intervals. In only one case, TT Aur, the course of the period changes appears more complex but the cyclic term and its parameters can be well explained in terms of LITE. This detailed analysis brought further evidences that the period changes in ET-systems really have significantly less complicated course than in LL-Algols and the finding of Hall (1989) was thus confirmed. These results, that is absence of alternate period changes in ET-systems, strengthen the evidence for an important role of the outer convective layer of a component of a binary in the period changes of LL-Algols. 
Table 5. Summary of changes of the orbital periods in the binaries with purely early-type components. The rate of change of the orbital period $\Delta P / P$ is given in days ${ }^{-1}$. Significance $S$ of the period change is defined in the text; the larger $S$, the better defined variation

\begin{tabular}{lcl}
\hline Binary & $S$ Period changes \\
\hline V337 Aql & 1.90 Decrease of period? \\
SX Aur & 3.19 Period increases: parabolic course $\Delta P / P=1.3610^{-10}$ \\
TT Aur & 1.37 Periodic term with $P_{2}=12.2$ yr and secular increase with parabolic course $\Delta P / P \approx 1.210^{-10}$ \\
BF Aur & 2.25 Period increases: parabolic course $\Delta P / P=1.7510^{-10}$ \\
AQ Cas & 1.02 Period constant \\
XZ Cep & 2.21 Period increases: parabolic course $\Delta P / P=3.2210^{-9}$ \\
V 448 Cyg & 1.11 Period constant \\
u Her & 1.07 Period constant \\
VY Lac & 1.00 Period constant \\
$\beta$ Lyr & $\quad$ Period increases: parabolic course $\Delta P / P=5.99710^{-7}$ (Harmanec \& Scholz 1993) \\
DM Per & 1.08 Period constant \\
IZ Per & 1.05 Period constant \\
RY Sct & 1.79 Period decreases: parabolic course $\Delta P / P=-3.7110^{-8}$ \\
RZ Sct & 1.49 Possible increase of period \\
V356 Sgr & $\quad$ Period constant? (Wilson \& Woodward 1995$)$ \\
Z Vul & 4.84 Period increases: parabolic course $\Delta P / P=2.8710^{-10}$ \\
\hline &
\end{tabular}

The magnitudes of the $\mathrm{O}-\mathrm{C}$ changes of ET-systems are usually less than one tenth of day. It cannot be therefore completely ruled out at present that the observed course of the $\mathrm{O}-\mathrm{C}$ changes in a particular ET-system (apart from the extreme variations in $\beta$ Lyr and RY Sct) is a consequence of LITE with so long period that the available data cover just a part of the cycle. Nevertheless, the common characteristics of the $\mathrm{O}-\mathrm{C}$ variations speak rather in favour of the real "intrinsic" changes in the prevailing majority of ET-systems analysed here.

There were accumulated evidences that many period changes in LL-Algols have character of episodes of abrupt changes (completed within less than a year) which are separated by intervals several years long during which the period length remains almost constant (e.g. U Cep Olson et al. 1981, RR Dra - Pokorný \& Zlatuška 1976). The typical value of the period change in a single episode in LL-Algols is $\triangle P / P \approx 10^{-5}$ days $^{-1}$. These episodes can be even grouped into seasons of enhanced activity (RW Tau - Šimon 1997c) and the underlying mechanism may be found in the structure changes of the late-type loser with COL. Unfortunately, the available data analysed here do not resolve the character of the course of the period changes in ET-systems in detail. The possibility of abrupt change was discussed in several ET-systems in the present analysis (BF Aur, Z Vul, XZ Cep, SX Aur). The data would suggest only one episode inside the interval several decades long. Nevertheless, the abrupt changes are hardly resolvable from the smooth course in the available data. The polynomial fits to the $\mathrm{O}-\mathrm{C}$ values of the respective ET-systems have shown that even the most variable periods could be plausibly matched by the second order polynomials and can be treated as smooth. We can only state that if these changes are really abrupt then the typi- cal corresponding value of $\Delta P / P$ is about $210^{-6}$ days $^{-1}$, that is lower than in LL-Algols.

Now we will turn to the mechanisms which can lead to the monotonic period changes in ET-systems and search for their possible contribution to the observed variations. Two basic groups of mechanisms can be established: (i) mass transfer and/or mass loss from the system; (ii) changes of the rotational angular momenta of the components.

As the semi-detached configuration implies these ETsystems are a result of the mass exchange and this transfer still continues at least in some of them. The binary begins its mass transfer epoch with $q>1$ and its value decreases in the course of the subsequent evolution. The models show that the largest $\dot{m}$ occurs in the early phase of the transfer and decreases afterwards. It means that less evolved systems should in principle possess larger period changes. As was shown above (Figs. 18 and 17) this trend is really apparent in the close systems with $P<7$ days.

Another less numerous group of wider systems with significantly longer orbital periods $(\beta$ Lyr, RY Sct and AQ Cas) can be resolved in Fig. 17ab. Two of these systems, $\beta$ Lyr and RY Sct, possess the largest absolute magnitudes of the period changes in the set analysed here (Fig. 18), despite of their low values of $q \approx 0.3$. In the same way, these two binaries display very strong photometric and spectroscopic activity connected with the large-scale mass transfer. This transfer gives rise to a huge accretion disk completely veiling the gainer in $\beta$ Lyr (and probably also in RY Sct). The high value of $\dot{m}$ in $\beta$ Lyr, determined from the period changes by Harmanec \& Scholz (1993), acceptably agrees with that determined from the model of the disk (Hubený \& Plavec 1991) and the connection of the period change with the mass transfer appears to be well established. The huge mass loss rate from RY Sct 
determined from the radio data is $\dot{m}_{\text {loss }}=610^{-5} M_{\odot} \mathrm{yr}^{-1}$ (Milano et al. 1981). RY Sct is the only one system of this set in which the period decrease is clearly present. An explanation for this decrease can be offered in terms of the intermediate mode (Huang 1963) when the outflowing matter forms a circumbinary ring removing angular momentum from the system. In this case it is necessary to find a mechanism which forces the matter to escape from the binary and form the ring. The radiative force of the early-type components is worthy consideration. The evaluation of the influence of this force on the Roche geometry of the respective ET-systems was carried out using the approach of Huang \& Taam (1990). They made use of the coefficients $a_{1}, a_{2}$ which characterize the efficiency of the radiative force with respect to the gravity. Values of $a_{1}, a_{2}$ are in the range from 0 (no radiative force) to 1 (radiative force just balances the gravity force). Although the uncertainty of the parameters of RY Sct may be appreciable the values of $a_{1}, a_{2}$ for both stars of this system are several tenths, which is more than an order of magnitude larger than for the rest systems (with the exception of V 448 Cyg and XZ Cep). As can be seen from the model by Huang $\&$ Taam (1990) such a large value of $a_{1}, a_{2}$ may alter the Roche geometry. In an extreme case one of the lobes can open while the $L_{1}$ point is secured. Although Huang and Taam argued that the effect of radiation becomes reduced in the case when a star occupy a large part of its lobe the role of the exceptionally strong radiative field in RY Sct seems to offer at least a tentative explanation for the large mass loss rate and consequently the rate of the period decrease observed.

As for AQ Cas, its physical and geometric parameters are very similar to $\beta$ Lyr but its activity is significantly lower. Both stars of AQ Cas are directly visible (Olson 1994) and there is not any sign of a huge accretion disk. The constant period of AQ Cas can be then explained by a much smaller $\dot{m}$ in comparison with $\beta$ Lyr.

The above mentioned difference between the groups of the short and long-period ET-systems can be clarified if the different evolutionary modes are taken into account. Case A operates for systems with the initial periods of the order of a day while wider binaries evolve through case B. As the models show (e.g. Pols 1994) the evolution through case B leads to a lower value of $q$ than in case A and, moreover, since the duration of the mass transfer epoch for case B is significantly shorter than in case A it will give rise to higher mass transfer rate and consequently a larger period change can be expected. The analysis of the evolutionary status is available for several ET-systems. SX Aur, DM Per, u Her are probably products of case A while $\beta$ Lyr, RZ Sct, V 356 Sgr (and possibly Z Vul) evolve through case B (Giuricin \& Mardirossian 1981b). The evolution of $\beta$ Lyr and V 356 Sgr through case B was also confirmed by the models of Ziolkowski (1976 and 1985). Harries et al. (1997) preferred case B for XZ Cep and
V 448 Cyg. When these evolutionary scenarios are compared with the diagrams in Figs. 17 and 18 we find that the only systems evolving through case $\mathrm{A}$ in which the period changes are detected are binaries with still quite high $q$ (SX Aur and TT Aur). The rest evolved systems possessing period change are of case B ( $\beta$ Lyr, XZ Cep, $\mathrm{Z} \mathrm{Vul).}$

Not only the mass ratio but also the mass of the loser plays a role in the period changes. It can be inferred from the densely populated interval of masses $1.5-8 M_{\odot}$ in the mass-luminosity diagram (Fig. 19). The systems (apart from BF Aur) are after the phase of rapid mass transfer (because of their clearly reversed $q$ ) and the luminosity of the losers is therefore expected to grow again. These stars then move in an approximately parallel direction in Fig. 19, i.e. towards smaller masses and higher luminosities. The distribution of systems in this figure may therefore reflect a division between the evolution of the losers of lower and higher mass to some extent. The systems with the components of higher mass evolve faster and possess higher $\dot{m}$.

In the light of the evidences discussed above it is tempting to understand the observed period changes in ET-systems as consequences of the mass transfer. If the period in the evolved systems with $q<1$ is variable then we observe its increase in those systems in which the radiative forces are negligible. In this interpretation the increase of $P$ may be reconciled with the dominant conservative mode. A possible contribution of the Jeans mode (matter escaping from the system at a high speed; Huang 1963) cannot be separated since it prolongs the orbital period, too. The dominant intermediate mode, leading to the period decrease, is supposed only in the high-luminosity RY Sct in which the radiative forces are strong.

However, the case of the period change in BF Aur, although well documented, cannot be decided at present. The available solutions do not give unique configuration and it is not possible to identify the loser and gainer unambiguously. It cannot be even ruled out that BF Aur is still before the onset of the mass transfer epoch. In the framework of the model of Demircan et al. (1997) the increase of the period length may be attributed to the dominant conservative mode. Since the value of the observed $\Delta P / P$ in this mode goes to zero as $q \rightarrow 1$ then even high $\dot{m}$ can lead just to marginal period change in BF Aur.

Although possible colliding winds in BF Aur were inferred from the photometry by Kallrath \& Kamper (1992) they are unlikely to be the cause of the observed change since even for the pure Jeans mode very large $\dot{m}_{\text {loss }} \approx$ $210^{-7} M_{\odot} \mathrm{yr}^{-1}$ would be needed.

It is interesting to determine at least an order of magnitude value of the mass transfer rate for the conservative case (which allows to keep the number of free parameters as low as possible) to get an idea of $\dot{m}$ which can be derived from the observed O-C changes (Huang 1963). 
Table 6. The mass transfer rate determined from the observed period changes assuming the conservative mode

\begin{tabular}{|c|c|}
\hline Binary & $\dot{m}\left(M_{\odot} \mathrm{yr}^{-1}\right)$ \\
\hline SX Aur & $1.710^{-7}$ \\
\hline TT Aur & $1.810^{-7}$ \\
\hline XZ Cep & $8.310^{-7}$ \\
\hline$\beta$ Lyr & $3.410^{-5}$ \\
\hline $\mathrm{Z} \mathrm{Vul}$ & $5.710^{-8}$ \\
\hline
\end{tabular}

The rate of the period change $\Delta P / P$ for this mode can be expressed as

$\frac{\Delta P}{P}=\frac{-3 \dot{m}_{\mathrm{l}}\left(M_{\mathrm{g}}-M_{\mathrm{l}}\right)}{M_{\mathrm{l}} M_{\mathrm{g}}}$

where $M_{\mathrm{g}}$ and $M_{\mathrm{l}}$ are masses of the gainer and loser, respectively, and $\dot{m}_{1}$ denotes the mass transfer rate per orbital cycle. The values of $\dot{m}$ in $M_{\odot} \mathrm{yr}^{-1}$ for the evolved systems $(q<1)$ which display period increase were obtained using Eq. (14) and are given in Table 6. It can be seen that the lower limit for which the period change can be recognized from the data is about $610^{-8} M_{\odot} \mathrm{yr}^{-1}$. Such a value is consistent with the phase of slow mass transfer and implies that generally the period changes of this magnitude, corresponding to the advanced evolutionary phase of a system, should be still revealed if they were indeed present. A direct comparison of the values from Table 6 with $\dot{m}$ determined from the observations of the circumstellar matter is difficult because as the position in the $r-q$ diagram suggests there is only little room left in the lobe of the gainer in most these systems (apart from $\beta$ Lyr-see above). It should be noted that the period changes produced by the mass transfer rates obtained here (apart from $\beta$ Lyr) would be easily overlapped by the activity of the late-type component in LL-Algols.

Two systems, AQ Cas and RZ Sct, for which the mass transfer rates $\dot{m}$ (about $510^{-7} M_{\odot} \mathrm{yr}^{-1}$ ) determined by Olson \& Bell (1989) from the photometric manifestations of the mass stream are available enabled a comparison of the expected period changes with the observed ones. The conservative transfer was used for this comparison (Figs. 6 and 14) and although this approach is an idealization it is useful as the first step since it does not require free parameters. The $\mathrm{O}-\mathrm{C}$ values of $\mathrm{AQ}$ Cas are consistent with the constant period (within the scatter) while the corresponding increase of the period length is possible in RZ Sct. Nevertheless, the scatter of the available timings precludes definite conclusion and new photoelectric data are urgently needed here.

The influence of the magnetic activity which is supposed to be strong in cool stars with COL and which can be the cause of the alternate period changes (Hall 1989; Applegate 1992) is missing in ET-systems. Also differences of the internal structure of the late and early-type stars can play a role because they affect the constant of the apsidal motion $k_{22}$. Changes of $k_{22}$ can give rise to variations of $P$ (Matese \& Whitmire 1983) and the larger value of $k_{22}$, the larger its contribution to the possible period changes. $k_{22}$ is very sensitive to the density profile $\rho(r)$. As the calculations of the contributions of the respective parts of a star to $k_{22}$, presented by Petrova (1995), showed $k_{22}$ is governed namely by the layers within which 0.9 of the total mass of the star is contained. The decrease of the density with the radius in the radiative outer layers of the early-type stars is more rapid than in the late-type stars with COL (to which the losers in LL-Algols belong). It implies that 0.9 mass is contained within a significantly smaller fractional radius in the early-type stars and hence these stars possess smaller $k_{22}$ in comparison with the latestars having COL. While even the outer layers can affect $k_{22}$ in late-type stars, these layers, supposed to be most influenced by the mass loss or accretion, contribute little to $k_{22}$ of the early-type stars. The components of ET-systems therefore should be more similar to the point-like masses. Moreover, radiative outer layers can react only on the Kelvin-Helmholtz time scale $\left(\approx 10^{5}\right.$ years $)$. Fluctuations in the outer layer of a star, such as those supposed for the late-type loser in RW Tau by Šmon (1997c), are then unlikely to produce the observed variations of the periods in ET-systems. These arguments bring another support to the interpretation of the period changes in ET-systems in terms of the mass transfer processes.

An additional mechanism which can play a role in the period changes of ET-systems is a conversion of the orbital angular momentum (OAM) into rotational angular momentum of the gainer (RAM) during the mass exchange, as was treated by Wilson \& Stothers (1975). The oblique impact of the stream can spin-up the gainer and increase thus RAM of this star. This value is removed from OAM. This process will therefore tend to decrease the length of the orbital period. We can judge the efficiency of this mechanism from the position of the binary in the $r-q$ diagram. The stream strikes the gainer almost perpendicularly to its photosphere in systems with high $r=R_{\mathrm{g}} / a$ and does not spin-up this star significantly. Moreover, the tidal coupling in such close systems is strong and will effectively synchronize the rotation of the gainer. As can be seen in Fig. 20 the degree of the asynchronous rotation in ET-systems of this set generally grows with decreasing $r$ and begins to be appreciable for systems with $r$ smaller than about 0.3 . We can conclude that while the contribution of this mechanism is small for close systems with $r>0.3$ the situation is different in wider binaries (namely AQ Cas, V 356 Sgr) since the impact of the stream is almost tangential (Fig. 17a) and the spinning-up very efficient (Fig. 20). The magnitude of the period changes can be therefore considerably diminished. The efficiency of this conversion is about $80 \%$ in case of AQ Cas, it means that the remaining period change inferred from the photometric determination of $\dot{m}$ (Olson \& Bell 1989) can be then 
completely hidden in the scatter of the data (Fig. 6). The conversion is even larger in the case of V 356 Sgr.

The influence of this conversion is further amplified in the systems which are in an advanced state of evolution and have thus small $q$. As was demonstrated by Ureche (1976) the ratio of RAM to OAM increases for $q$ significantly different from unity, it means that the role of the rotational momentum of the components becomes appreciable here, namely if the rotation of the gainer is highly asynchronous.

Acknowledgements. This research has made use of the international database of timings and I thank F. Agerer for providing me with the data. I also thank Dr. Z. Mikulášek for reading the manuscript and for the comments. This study was supported by the project K1-003-601/4 Astrophysics of non-stationary stars of the Academy of Sciences of the Czech Republic.

\section{References}

Agerer F., 1995 (private communication)

Agerer F., 1996 (private communication)

Alduseva V.Ya., 1977, Perem. Zvezdy 20, 375

Applegate J.H., 1992, ApJ 385, 621

Bell S.A., Adamson A.J., Hilditch R.W., 1987, MNRAS 224, 649

Biermann P., Hall D.S., 1973, A\&A 27, 249

Catalano F.A., Catalano S., Rodono M., 1971, Ap\&SS 11, 232

Danielkiewicz-Krosniak E., Kurpinska-Winiarska M., 1996, Rocznik astronomiczny Observatorium Krakowskiego, No. 67 (SAC96)

De Greve J.P., 1993, A\&AS 97, 527

de Martino D., Vittone A.A., Rossi C., Giovannelli F., 1992, A\&A 254, 266

Demircan O., Ozdemir S., Albayrak B., Ak H., Tanriver M., Degirmenci O.L., Erdem A., Sezer C., 1997, Rev. Mex. Astron. Astrofis. 33, 131

Djurasevic G., Demircan O., Ozdemir S., Tanriver M., Ak H., Muyesseroglu Z., Albayrak B., 1997, Ap\&SS (submitted)

Dugan R.S., Wright F.W., 1939, Princeton Contrib. 19, 52

Eaton J.A., 1978, Acta Astron. 28, 601

Etzel P.B., Olson E.C., 1993, AJ 106, No. 3, 1200

Figueiredo J., De Greve J.P., Hilditch R.W., 1994, A\&A 283, 144

Giuricin G., Mardirossian F., 1981a, A\&AS 45, 499

Giuricin G., Mardirossian F., 1981b, ApJSS 46, 1

Glazunova L.V., Karetnikov V.G., 1985, Astron. Zh. 62, 938

Hall D.S., 1989, Space. Sci. Rev. 50, 219

Hall D.S., 1991, ApJ 380, L85

Hansen H.K., McNamara D.H., 1959, ApJ 130, 791

Hanžl D., 1994, IBVS No. 4097

Harmanec P., 1988, Bull. Astron. Inss. Czechosl. 39, 329

Harmanec P., 1990, A\&A 237, 91

Harmanec P., Scholz G., 1993, A\&A 279, 131

Harmanec P., Morand F., Bonneau D., Jiang Y., Yang S., Guinan E.F., Hall D.S., Mourard D., Hadrava P., Božić H., Sterken C., Tallon-Bosc I., Walker G.A.H., McCook G.P., Vakili F., Stee Ph., Le Contel J.M., 1996, A\&A 312, 879

Harries T.J., Hilditch R.W., Hill G., 1997, MNRAS 285, 277

Hilditch R.W., Hill G., 1975, MNRAS 172, 29
Hilditch R.W., Bell S.A., 1987, MNRAS 229, 529

Huang Su-Shu, 1963, ApJ 138, 471

Huang R.Q., Taam R.E., 1990, A\&A 236, 107

Hubený I., Plavec M.J., 1991, AJ 102, 1156

Kalimeris A., Rovithis-Livaniou H., Rovithis P., 1994, A\&A 282,775

Kallrath J., Kamper B.-C., 1992, A\&A 265, 613

King A.R., Jameson R.F., 1979, A\&A 71, 326

Koch R.H., Plavec M., Wood F.B., 1970, Publ. Univ. Pennsylvania, Astron. Ser., Vol. 11

Kovachev B.J., Reinhardt M., 1975, Acta Astron. 25, 133

Kreiner J.M., Kumsiashvili M.I., Tremko J., 1990, Bull. Astron. Inss. Czechosl. 41, 51

Kreiner J.M., Tremko J., 1978, Acta Astron. 28, 179

Kreiner J.M., Ziolkowski J., 1978, Acta Astron. 28, 497

Kreiner J.M., Tremko J., 1993, New Frontiers in Binary Star Research, ASP Conf. Ser. 38, 351

Leung K.C., 1989, Space. Sci. Rev. 50, 279

Lubow S.H., Shu F.H., 1975, ApJ 198, 383

Lucy L.B., Sweeney M.A., 1971, AJ 76, 544

Mammano A.M., Margoni R., Stagni R., 1974, A\&A 35, 143

Matese J.J., Whitmire D.P., 1983, A\&A 117, L7

McNamara D.H., 1957, PASP 69, 574

Milano L., Vittone A., Ciatti F., Mammano A., Margoni R., Strazzulla G., 1981, A\&A 100, 59

Mayer P., 1987, Bull. Astron. Inss. Czechosl. 38, 58

Mayer P., 1990, Bull. Astron. Inss. Czechosl. 41, 231

Olson E.C., 1994, AJ 108, 666

Olson E.C., Crawford R.C., Hall D.S., Louth H., Markworth N.L., Piirola V., 1981, PASP 93, 464

Olson E.C., Bell D.J., 1989, PASP 101, 907

Olson E.C, Ezel P., 1994, AJ 108, 262 (OE94)

Paczynski B.E., 1967, Acta Astron. 17, 355

Peters G.J., 1989, Space. Sci. Rev. 50, 9

Petrova A.V., 1995, AZh 72, No. 6, 924

Plavec M., 1968, Adv. Astr. Astroph. 6, 201

Plavec M.J., Dobias J.J., Etzel P.B., Weiland J.L., 1984, in Future of UV Astronomy Based on Six Years of IUE Research, NASA CP-2349, Mead J.M., Chapman R.D., Kondo Y. (eds.), p. 420

Pokorný Z., Zlatuška J., 1976, Bull. Astron. Inss. Czechosl. 27, 341

Polidan R.S., 1989, Space. Sci. Rev. 50, 85

Pols O.R., 1994, A\&A 290, 119

Popper D.M., 1980, Ann. Rev. Astron. Astrophys. 18, 115

Richman H.R., Applegate J.H., Patterson J., 1994, PASP 106, 1075

Sahade J., Wood F.B., 1978, Interacting Binary Stars. Pergamon Press, p. 142

Semeniuk I., Kaluzny J., 1984, Acta Astr. 34, 207

Skulskij M. Yu., 1992, AZh 69, 803

Stellingwerf R.F., 1978, ApJ 224, 953

Stothers R., 1973, PASP 85, 363

Šimon V., 1996, A\&A 311, 915

Simon V., 1997a, The Proceedings of the $28^{\text {th }}$ Conference on Variable Star Research, Brno, Czech Republic, p. 106

Simon V., 1997b, The Proceedings of the $28^{\text {th }}$ Conference on Variable Star Research, Brno, Czech Republic, p. 112

Šimon V., 1997c, A\&A 319, 886

Ureche V., 1976, IAU Symposium No. 73, Structure and Evolution of Close Binary Systems, Eggleton P., Mitton S., Whelan J. (eds.), p. 351 
Van Hamme W., Wilson R.E., 1990, AJ 100, No. 6, 1981

Volkova G.V., Glazunova L.V., Tarasov A.E., 1993, AZh 70, No. 1, p. 91

Wilson R.E., 1974, ApJ 189, 319

Wilson R.E., Stothers R., 1975, MNRAS 170, 497

Wilson R.E., Caldwell C.N., 1978, ApJ 221, 917

Wilson R.E., Woodward E.J., 1995, PASP 107, No. 708, 132

Wolf G.W., West S.R.D., 1993, New Frontiers in Binary Star Research, ASP Conf. Ser. 38, 312
Wood F.B., 1950, ApJ 112, 196

Yavuz I., 1969, A\&A 2, 388

Zakirov M.M., 1992, Kinem. Fiz. Nebes. Tel. 8, No. 3, p. 14

Zhang R., Kim J., Zhang J., Zhang X., Kang Y.W., Zhai D., 1993, IBVS, No. 3941

Ziolkowski J., 1976, ApJ 204, 512

Ziolkowski J., 1985, Acta Astron. 35, 199

Zola S., 1995, A\&A 294, 525 